\title{
MERCURY AUDIT AT ROCKY MOUNTAIN ARSENAL
}

\author{
S. M. Smith \\ M. K. Jensen \\ G. M. Anderson* \\ *Rocky Mountain Arsenal \\ DATE PUBLISHED - FEBRUARY 1994
}

\author{
Prepared for the \\ HAZWRAP SUPPORT CONTRACTOR OFFICE \\ Oak Ridge, Tennessee \\ Prepared by \\ OAK RIDGE NATIONAL LABORATORY \\ Oak Ridge, Tennessee 37831-6036 \\ managed by \\ MARTIN MARIETTA ENERGY SYSTEMS, INC. \\ for the \\ U.S. DEPARTMENT OF ENERGY \\ under Contract DE-ACO5-840R21400
}




\section{CONTENTS}

TABLES $\ldots \ldots \ldots \ldots \ldots \ldots \ldots \ldots \ldots \ldots \ldots \ldots \ldots \ldots$

FIGURES $\ldots \ldots \ldots \ldots \ldots \ldots \ldots \ldots \ldots \ldots \ldots \ldots \ldots \ldots$ v

EXECUTTVE SUMMARY .................... vii

1. INTRODUCTION $\ldots \ldots \ldots \ldots \ldots \ldots \ldots \ldots \ldots \ldots \ldots \ldots$

1.1 BACKGROUND $\ldots \ldots \ldots \ldots \ldots \ldots \ldots \ldots \ldots \ldots \ldots$

1.2 TASK DESCRIPTION $\ldots \ldots \ldots \ldots \ldots \ldots \ldots \ldots \ldots \ldots$

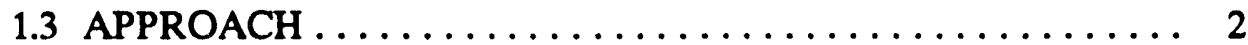

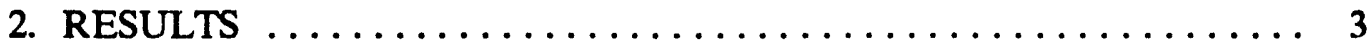

2.1 MERCURY AUDIT RESULTS $\ldots \ldots \ldots \ldots \ldots \ldots \ldots \ldots \ldots$

2.1.1 Results from Buildings and Tanks Audited ....... 3

2.1.2 Other Buildings and Tanks $\ldots \ldots \ldots \ldots \ldots \ldots \ldots 3$

2.2 DETECTIONS ........................ 28

2.2.1 Visible Mercury Spills . . . . . . . . . . . 28

2.2 .2 Other Detections $\ldots \ldots \ldots \ldots \ldots \ldots \ldots \ldots \ldots \ldots$

2.3 INSTRUMENTATION $\ldots \ldots \ldots \ldots \ldots \ldots \ldots \ldots \ldots \ldots \ldots$

3. HEALTH AND SAFETY ISSUES $\ldots \ldots \ldots \ldots \ldots \ldots \ldots \ldots \ldots \ldots$

REFERENCES $\ldots \ldots \ldots \ldots \ldots \ldots \ldots \ldots \ldots \ldots \ldots \ldots \ldots \ldots \ldots \ldots$

ACRONYMS AND INITIALISMS $\ldots \ldots \ldots \ldots \ldots \ldots \ldots \ldots \ldots \ldots$

APPENDIX A: MERCURY AUDIT DATA BASE 


\section{TABLES}

2.1 Mercury audit results $\ldots \ldots \ldots \ldots \ldots \ldots \ldots \ldots \ldots \ldots \ldots \ldots$

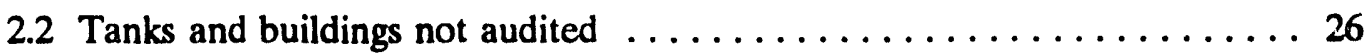

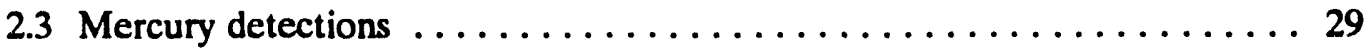

\section{FIGURES}

1. Front view of instrument control panel and rear view of mercury pot $\ldots \ldots 31$

2. Mercury-containing manometer $\ldots \ldots \ldots \ldots \ldots \ldots \ldots \ldots \ldots \ldots \ldots \ldots \ldots \ldots$

3. Mercoid controls and Mercoid thermostat ............... 34

4. Honeywell ${ }^{m}$ chart recorder with inside view of mercury bulbs . . . . . 35

5. Panalarm ${ }^{n}$ gauge, front and rear view $\ldots \ldots \ldots \ldots \ldots \ldots \ldots \ldots \ldots$

6. Honeywellim Pressuretrol ${ }^{\mathrm{m}}$ switch with mercury bulb $\ldots \ldots \ldots \ldots$

7. Honeywell ${ }^{m}$ Pressuretrol ${ }^{m}$ switch and Honeywell ${ }^{m M}$ gas pressure switch .. 39 


\section{EXECUTIVE SUMMARY}

This report presents the results of an environmental compliance audit to identify potential mercury-containing equipment in 261 buildings and 197 tanks at the Rocky Mountain Arsenal (RMA). The RMA, located near Denver, Colorado, is undergoing clean up and decommissioning by the Department of the Army. Part of the decommissioning procedure is to ensure that all hazardous wastes are properly identified and disposed of. The purpose of the audit was to identify any mercury spills and mercury-containing instrumentation.

The audits were conducted from April 7, 1992, through July 16, 1992, by a two-person team. The team interviewed personnel with knowledge of past uses of the buildings and tanks. Information concerning past mercury spills and the locations and types of instrumentation that contain mercury proved to be invaluable for an accurate survey of the arsenal. The team used a Jerome $431-\mathrm{X}^{\mathrm{m}}$ Mercury Vapor Analyzer to detect spills and confirm locations of mercury vapor. Twelve detections were recorded during the audit and varied from visible mercury spills to slightly elevated readings in the corners of rooms with past spills. The audit also identified instrumentation that contained mercury. All data have been incorporated into a computerized data base that is compatible with the RMA data base. 


\section{INTRODUCTION}

\subsection{BACKGROUND}

The Rocky Mountain Arsenal (RMA), which has been used for the production of a wide variety of chemical compounds for both military and commercial applications, is under the control of the Department of the Army. The Department of the Army is in the process of cleaning up and decommissioning the RMA, and as part of that deactivation procedure, all hazardous wastes are to be properly identified and disposed of. Interim remedial actions are presently underway, and the installation is listed on the Environmental Protection Agency's (EPA) National Priority List (NPL).

To obtain assistance for environmental compliance and decommissioning activities, the Program Manager Rocky Mountain Arsenal (PMRMA) entered into an interagency agreement with the Department of Energy (DOE). The Hazardous Waste Remedial Actions Program (HAZWRAP), a division of Martin Marietta Energy Systems, Inc., a DOE contractor, was tasked to provide that assistance. HAZWRAP's support to PMRMA is defined in the program management plan (HAZWRAP 1991). HAZWRAP assigned the task of auditing for mercury to Oak Ridge National Laboratory (ORNL), to be performed by ORNL's Grand Junction, Colo., office. A work plan was prepared to accomplish this task (ORNL 1992).

\subsection{TASK DESCRIPTION}

The audit was conducted during three trips to the RMA during April, May, and July 1992. The list of buildings and tanks audited was supplied by PMRMA with the objective to locate any mercury spills and to identify instrumentation containing mercury. Locations of mercury, detection of spills, readings, and 
comments were recorded on field sheets during a walk-through survey of each building and around each tank. Analyzer readings were taken in every room of each building and at all instrumentation; comments were entered on the field sheets if a building or tank could not be surveyed. This information was to be made available in electronic format to the RMA data base for review before any remedial action took place. A description of the ORNL data base appears in Appendix A.

\subsection{APPROACH}

The Jerome 431- $\mathrm{X}^{\mathrm{TM}}$ Mercury Vapor Analyzer was selected for this audit because it is portable and the gold film sensor is inherently stable and selective to mercury, thus eliminating interferences (such as water vapor and hydrocarbons) that are common to ultraviolet analyzers. The instrument's detection range is 0.003 to $0.999 \mathrm{mg} / \mathrm{m}^{3}$, with a precision of $5 \%$ relative standard deviation at $0.100 \mathrm{mg} / \mathrm{m}^{3}$ of mercury.

Because of its low vapor pressure $(0.0012 \mathrm{~mm})$, mercury is not easily detected when left undisturbed. Since mercury tends to settle in cracks and corners, it becomes more difficult to detect over a period of time both visually and with a mercury vapor analyzer due to dust and oxidation.

Given these characteristics of mercury, the audit team began its investigation by meeting with PMRMA personnel familiar with plant activities. During these interviews, the team gathered information about past and present mercury spills, equip-ment, laboratory uses, and locations where mercury may have been used. Health and safety concerns were also discussed. Information obtained during these interviews made locating mercury spills much easier. 


\section{RESULTS}

The results of the audit are presented in two categories: (1) the results of the comprehensive audit that was structured to identify the presence of all mercury in and around the buildings and tanks, and (2) the detection of all spilled or unconfined mercury in any of these areas. The first category is referred to as the mercury audit results and the second as detections.

\section{MERCURY AUDIT RESULTS}

\subsection{Results from Buildings and Tanks Audited}

Audit results for buildings and tanks are presented in Table 2.1, with building numbers and tank numbers listed alphanumerically. Detection numbers, analyzer readings, and locations identify all detections. The comments section lists the known mercury-containing or possibly mercury-containing instruments and their locations. Any building not audited or locations where only partial audits were performed are also noted in the comments section.

\subsection{Other Buildings and Tanks}

Twenty-five buildings from the original list were not audited for one of three reasons: 1) the building no longer exists or could not be located; 2) access to the building was denied due to physical barriers, for example locked doors, boarded up buildings, and fenced off areas; or 3 ) unsafe conditions prevented auditing. A list of unaudited buildings is presented in Table 2.2 . 
Table 21. Mercury audit results

\begin{tabular}{|c|c|c|c|c|c|}
\hline $\begin{array}{l}\text { Building } \\
\text { No. }\end{array}$ & $\begin{array}{l}\text { Tank } \\
\text { No. }\end{array}$ & $\begin{array}{l}\text { Detection } \\
\text { No. }\end{array}$ & $\begin{array}{c}\text { Analyzer } \\
\text { Reading, } \\
\mathrm{mg} / \mathrm{m}^{3} \mathrm{Hg}\end{array}$ & $\begin{array}{l}\text { Location of } \\
\text { Detection }\end{array}$ & Comments \\
\hline 111 & & 0 & & & \\
\hline 112 & & 0 & & & \\
\hline $112 \mathrm{~A}$ & & 0 & & & \\
\hline 135 & & 0 & & & \\
\hline 143 & & 0 & & & \\
\hline 145 & & 0 & & & \\
\hline 211 & & 0 & & & \\
\hline 213 & & 0 & & & \\
\hline 241 & & 1 & 0.128 & $\begin{array}{l}\text { Old laboratory } \\
\text { room }\end{array}$ & $\begin{array}{l}\text { Elevated readings inside the center } \\
\text { lab bench and the northernmost } \\
\text { bottom cabinet, west side. The } \\
\text { cabinet is marked asbestos (no } \\
\text { visible mercury). }\end{array}$ \\
\hline 242 & & 0 & & & \\
\hline 243 & & 1 & 0.010 & $\begin{array}{l}\text { East wall/ } \\
\text { switchroom/ } \\
\text { 2nd floor }\end{array}$ & $\begin{array}{l}\text { Elevated readings in the } \\
\text { switchroom ( } 2 \text { nd room from west } \\
\text { end) from an in-line pressure } \\
\text { gauge (manometer) containing } \\
\text { mercury. A pot that may contain } \\
\text { mercury was also found. }\end{array}$ \\
\hline 244 & & 0 & & & Building no longer exists. \\
\hline 245 & & 0 & & & \\
\hline 251 & & 0 & & & $\begin{array}{l}\text { Stairs to upper floor boarded off. } \\
\text { Checked ground floor only. }\end{array}$ \\
\hline 307 & & 0 & & & \\
\hline 309 & & 0 & & & \\
\hline 311 & & 0 & & & \\
\hline 312 & & 0 & & & \\
\hline 313 & & 0 & & & $\begin{array}{l}\text { Laboratory building; one lab was } \\
\text { used to store mercury in the past, } \\
\text { but no elevated readings were } \\
\text { measured during the audit. }\end{array}$ \\
\hline 314 & & 0 & & & \\
\hline 315 & & 0 & & & \\
\hline $315 \mathrm{~A}$ & & 0 & & & \\
\hline 319 & & 0 & & & \\
\hline
\end{tabular}


Table 2.1. (continued)

\begin{tabular}{|c|c|c|c|c|c|}
\hline $\begin{array}{l}\text { Building } \\
\text { No. }\end{array}$ & $\begin{array}{l}\text { Tank } \\
\text { No. }\end{array}$ & $\begin{array}{c}\text { Detection } \\
\text { No. }\end{array}$ & $\begin{array}{c}\text { Analyzer } \\
\text { Reading, } \\
\mathrm{mg} / \mathrm{m}^{3} \mathrm{Hg}\end{array}$ & $\begin{array}{l}\text { Location of } \\
\text { Detection }\end{array}$ & Comments \\
\hline 321 & & 1 & 0.764 & $\begin{array}{l}\text { North wally } \\
2 \text { nd floor }\end{array}$ & $\begin{array}{l}\text { 1) Behind the instrument panel, } \\
\text { visible spills of mercury were } \\
\text { detected on the floor. The } \\
\text { instruments still in use have } \\
\text { pots containing mercury. } \\
\text { 2) Throughout the building are } \\
\text { Mercoid switches still in use } \\
\text { containing small amounts of } \\
\text { mercury. }\end{array}$ \\
\hline $321 \mathrm{~A}$ & $321 \mathrm{~A}$ & 0 & & & \\
\hline $321 \mathrm{~B}$ & $321 \mathrm{~B}$ & 0 & & & \\
\hline $321 \mathrm{C}$ & & 0 & & & \\
\hline $321 \mathrm{D}$ & & 0 & & & \\
\hline $321 \mathrm{E}$ & $321 \mathrm{E}$ & 0 & & & \\
\hline 322 & & 0 & & & \\
\hline $322 \mathrm{~A}$ & & 0 & & & \\
\hline 325 & & 1 & 0.040 & $\begin{array}{l}\text { North wall/ } \\
\text { 1st floor }\end{array}$ & $\begin{array}{l}\text { Elevated readings of mercury were } \\
\text { detected on the floor behind the } \\
\text { control panel. Instruments on the } \\
\text { panel have pots containing } \\
\text { mercury. }\end{array}$ \\
\hline 325 & & 2 & 0.006 & $\begin{array}{l}\text { 2nd floor/ } \\
\text { southeast } \\
\text { corner }\end{array}$ & $\begin{array}{l}\text { Instrument assembly room was } \\
\text { used to drain mercury from old } \\
\text { meters. Slightly elevated readings } \\
\text { were recorded in the corner of the } \\
\text { room. }\end{array}$ \\
\hline 326 & & 0 & & & \\
\hline 328 & & 0 & & & $\begin{array}{l}\text { Mercoid switches found on 1st } \\
\text { floor, northeast corner of switch } \\
\text { gear room, on vertical piping. } \\
\end{array}$ \\
\hline $328 \mathrm{~A}$ & & 0 & & & \\
\hline 329 & & 0 & & & \\
\hline 331 & & 0 & & & \\
\hline 332 & & 0 & & & \\
\hline 333 & & 0 & & & \\
\hline 334 & & 0 & & & \\
\hline 335 & & 0 & & & \\
\hline 336 & & 0 & & & \\
\hline 337 & & 0 & & & \\
\hline 341 & & 0 & & & \\
\hline
\end{tabular}


Table 2.1. (continued)

\begin{tabular}{|c|c|c|c|c|c|}
\hline $\begin{array}{c}\text { Building } \\
\text { No. }\end{array}$ & $\begin{array}{l}\text { Tank } \\
\text { No. }\end{array}$ & $\begin{array}{c}\text { Detection } \\
\text { No. }\end{array}$ & $\begin{array}{l}\text { Analyzer } \\
\text { Reading, } \\
\mathrm{mg} / \mathrm{m}^{3} \mathrm{Hg} \\
\end{array}$ & $\begin{array}{l}\text { Location of } \\
\text { Detection }\end{array}$ & Comments \\
\hline $341 \mathrm{~A}$ & & 0 & & & \\
\hline $341 B$ & & 0 & & & \\
\hline 342 & & 0 & & & \\
\hline 343 & & 0 & & & \\
\hline $343 \mathrm{~A}$ & & 0 & & & Boarded up; could not access. \\
\hline 343B & & 0 & & & \\
\hline 344 & & 0 & & & \\
\hline 345 & & 0 & & & \\
\hline 346 & & 1 & 0.860 & Southeast wall & $\begin{array}{l}\text { A mercury spill was found in the } \\
\text { meter storage area. Small pools of } \\
\text { mercury are visible below an old } \\
\text { instrument. Additional mercury- } \\
\text { containing meters are stored in this } \\
\text { area. }\end{array}$ \\
\hline 346 & & 2 & 0.342 & $\begin{array}{l}\text { North wally } \\
\text { westernmost } \\
\text { door }\end{array}$ & $\begin{array}{l}\text { Two 5-gal buckets containing } \\
\text { approximately } 3 \text { gal of mercury in } \\
\text { separate plastic containers were } \\
\text { located near the northwest door. }\end{array}$ \\
\hline 351 & & 0 & & & \\
\hline $352 \mathrm{~A}$ & & 0 & & & \\
\hline 354 & & 0 & & & \\
\hline 355 & & 0 & & & \\
\hline 356 & & 0 & & & \\
\hline 361 & & 0 & & & \\
\hline 362 & & 0 & & & \\
\hline 364 & & 0 & & & \\
\hline 365 & & 0 & & & $\begin{array}{l}2 \text { Mercoid switches on east wall. } \\
\text { One is attached to the wall, and } \\
\text { one is on a table. }\end{array}$ \\
\hline 368 & & 0 & & & $\begin{array}{l}\text { The tank room of the swimming } \\
\text { pool has a level gauge that used } \\
\text { mercury. Also, bottles of mercury } \\
\text { were stored there in the past. No } \\
\text { elevated readings were detected } \\
\text { during the audit. }\end{array}$ \\
\hline 371 & & 0 & & & $\begin{array}{l}2 \text { Mercoid switches on the west } \\
\text { wall, 1st floor. } 2 \text { more Mercoid } \\
\text { switches on the west wall, basement } \\
\text { pump room. }\end{array}$ \\
\hline $372 A$ & & 0 & & & \\
\hline
\end{tabular}


Table 2.1. (continued)

\begin{tabular}{|c|c|c|c|c|c|}
\hline $\begin{array}{l}\text { Building } \\
\text { No. }\end{array}$ & $\begin{array}{l}\text { Tank } \\
\text { No. }\end{array}$ & $\begin{array}{l}\text { Detection } \\
\text { No. }\end{array}$ & $\begin{array}{l}\text { Anatyzer } \\
\text { Reading, } \\
\mathrm{mg} / \mathrm{m}^{3} \mathrm{Hg}\end{array}$ & $\begin{array}{l}\text { Location of } \\
\text { Detection }\end{array}$ & Comments \\
\hline 373 & & 0 & & & \\
\hline $373 B$ & & $\mathbf{0}$ & & & \\
\hline 378 & & $\mathbf{0}$ & & & $\begin{array}{l}\text { The old Building } 378 \text { on airport } \\
\text { property has been removed, and a } \\
\text { new Building } 378 \text { is now located } \\
\text { southeast of Building } 615 \text {. }\end{array}$ \\
\hline 379 & & 0 & & & \\
\hline 382 & & 0 & & & \\
\hline 383 & & 0 & & & \\
\hline$\overline{383 A}$ & & $\overline{0}$ & & & Building bas been removed. \\
\hline 385 & & 0 & & & \\
\hline 386 & & $\overline{0}$ & & & \\
\hline 387 & & 0 & & & \\
\hline 391 & & 0 & & & \\
\hline 392 & & 0 & & & \\
\hline 393 & & 0 & & & \\
\hline 409 & & 0 & & & \\
\hline 411 & & 0 & & & \\
\hline $411 \mathrm{~A}$ & & 1 & 0.480 & $\begin{array}{l}\text { Northeast } \\
\text { corner under } \\
\text { instrument }\end{array}$ & $\begin{array}{l}\text { Visible mercury spill from a } \\
\text { mercury pot in a recorder. }\end{array}$ \\
\hline $411 \mathrm{~B}$ & & 0 & & & \\
\hline 412 & & 0 & & & $\begin{array}{l}\text { Several Mercoid switches in the } \\
\text { storage room, northeast corner of } \\
\text { the building. }\end{array}$ \\
\hline 413 & & 0 & & & $\begin{array}{l}\text { Tank on the west side of the } \\
\text { building bas a mercury } \\
\text { thermometer on the north end. }\end{array}$ \\
\hline 425 & T0032 & 0 & & & \\
\hline 426 & R0019 & 0 & & & \\
\hline 428 & & 0 & & & \\
\hline 429 & & 0 & & & \\
\hline 431 & & 0 & & & \\
\hline 433 & & 1 & 0.024 & $\begin{array}{l}\text { South corri- } \\
\text { dor, south side } \\
\text { of building }\end{array}$ & $\begin{array}{l}2 \text { chant recorders are in the south } \\
\text { corridor, south side of building; no } \\
\text { visible mercury. }\end{array}$ \\
\hline $459 \mathrm{C}$ & & 0 & & & \\
\hline $462 A$ & $462 \mathrm{~A}$ & 0 & & & \\
\hline
\end{tabular}


Table 2.1. (continued)

\begin{tabular}{|l|l|c|c|c|l||}
\hline $\begin{array}{c}\text { Building } \\
\text { No. }\end{array}$ & $\begin{array}{c}\text { Tank } \\
\text { No. }\end{array}$ & $\begin{array}{c}\text { Detection } \\
\text { No. }\end{array}$ & $\begin{array}{c}\text { Analyzer } \\
\text { Reading, } \\
\mathrm{mg} \mathrm{m}^{3} \mathrm{Hg}\end{array}$ & $\begin{array}{c}\text { Location of } \\
\text { Detection }\end{array}$ & \\
\hline 463A & 463A & 0 & & & Comments \\
\hline $463 \mathrm{~B}$ & $463 \mathrm{~B}$ & 0 & & & \\
\hline $463 \mathrm{C}$ & $463 \mathrm{C}$ & 0 & & & \\
\hline $463 \mathrm{E}$ & $463 \mathrm{E}$ & 0 & & & \\
\hline $463 \mathrm{~F}$ & $463 \mathrm{~F}$ & 0 & & & \\
\hline $463 \mathrm{G}$ & $463 \mathrm{G}$ & 0 & & & \\
\hline $463 \mathrm{H}$ & $463 \mathrm{H}$ & 0 & & & \\
\hline 464 & & 0 & & & \\
\hline $464 \mathrm{~A}$ & $464 \mathrm{~A}$ & 0 & & & \\
\hline $464 \mathrm{~B}$ & $464 \mathrm{~B}$ & 0 & & & \\
\hline 471 & T0130 & 0 & & & \\
\hline 471 & T0131 & 0 & & & \\
\hline 471 & T0132 & 0 & & & \\
\hline 471 & T0133 & 0 & & & \\
\hline 471 & T0273 & 0 & & & \\
\hline 472 & T0208 & 0 & & & \\
\hline 473 & T1048 & 0 & & & \\
\hline 473 & T1142 & 0 & & & \\
\hline 473 & V1061 & 0 & & & \\
\hline 473 & V1064 & 0 & & & \\
\hline 512 & T1390 & 0 & & & \\
\hline 512 & T1391 & 0 & & & \\
\hline 512 & T1392 & 0 & & & \\
\hline 515 & R0038 & 0 & & & \\
\hline 515 & V1214 & 0 & & & \\
\hline 515 & V1220 & 0 & & & \\
\hline 522 & & 0 & & & \\
\hline $522 \mathrm{~B}$ & & 0 & & & \\
\hline 523 & & 0 & & & \\
\hline 527 & & 0 & & & \\
\hline
\end{tabular}


Table 2.1. (continued)

\begin{tabular}{|c|c|c|c|c|c|}
\hline $\begin{array}{l}\text { Building } \\
\text { No. }\end{array}$ & $\begin{array}{l}\text { Tank } \\
\text { No. }\end{array}$ & $\begin{array}{l}\text { Detection } \\
\text { No. }\end{array}$ & $\begin{array}{l}\text { Anatyzer } \\
\text { Reading, } \\
\mathrm{mg} / \mathrm{m}^{3} \mathrm{Hg}\end{array}$ & $\begin{array}{l}\text { Location of } \\
\text { Detection }\end{array}$ & Comments \\
\hline 536 & & 0 & & & $\begin{array}{l}\text { Located: } \\
\text { 1) } 9 \text { Mercoid meters in rear of } \\
\text { control panel in control room. } \\
\text { 2) } 1 \text { Mercoid switch on top of } \\
\text { vent line next to salt storage } \\
\text { tank. } \\
\text { 3) Foxborom pneumatic } \\
\text { transmitter next to salt storage } \\
\text { tank with a pot on back that } \\
\text { may contain mercury. } \\
\text { 4) } 2 \text { Mercoid switches on } 2 \text { nd } \\
\text { level on natural gas line in rear } \\
\text { of dryer tank. }\end{array}$ \\
\hline 537 & & 0 & & & $\begin{array}{l}\text { Located: } \\
\text { 1) Manometer containing } \\
\text { mercury in storage pen in } \\
\text { shipping W.T. room. } \\
\text { 2) Thermostat on cooling unit } \\
\text { inside middle door next to } \\
\text { caustic tank. } \\
\text { 3) Thermostat on cooling unit } \\
\text { behind mustard gas contain- } \\
\text { ment unit inside middle door } \\
\text { on north wall. } \\
\text { 4) Mercoid switch on compressor } \\
\text { behind middle mustard gas } \\
\text { containment unit inside middle } \\
\text { doors. }\end{array}$ \\
\hline 538 & & 0 & & & $\begin{array}{l}\text { Located: } \\
\text { 1) } 4 \text { Honeywellm temperature } \\
\text { control meters with mercury } \\
\text { bulbs on the south side of } \\
\text { north-central wall. } \\
\text { 2) Honeywellm temperature } \\
\text { control meter that may contain } \\
\text { mercury on control panel for } \\
\text { furnace. } \\
\text { 3) } 4 \text { Mercoid switches on control } \\
\text { panel in southeast comer, east } \\
\text { of furnace. } \\
\text { 4) Thermometer (400. F) above } \\
\text { and right of furnace control } \\
\text { panel. } \\
\text { 5) } 2 \text { Mercoid switches behind and } \\
\text { right of furnace control panel. }\end{array}$ \\
\hline $538 \mathrm{~A}$ & & 0 & & & $\begin{array}{l}7 \text { Mercoid switches on quench and } \\
\text { scrubber control panel. }\end{array}$ \\
\hline
\end{tabular}


Table 2.1. (continued)

\begin{tabular}{|c|c|c|c|c|c|}
\hline $\begin{array}{l}\text { Building } \\
\text { No. }\end{array}$ & $\begin{array}{l}\text { Tank } \\
\text { No. }\end{array}$ & $\begin{array}{l}\text { Detection } \\
\text { No. }\end{array}$ & $\begin{array}{l}\text { Analyzer } \\
\text { Reading, } \\
\mathrm{mg} / \mathrm{m}^{3} \mathrm{Hg}\end{array}$ & $\begin{array}{l}\text { Location of } \\
\text { Detection }\end{array}$ & Comments \\
\hline 539 & & 0 & & & \\
\hline 540 & & 0 & & & $\begin{array}{l}\text { Mercoid switch on a Moynom } \\
\text { pump near southeast corner. }\end{array}$ \\
\hline 541 & & 0 & & & $\begin{array}{l}\text { In bay "K" on north side of } \\
\text { building, a Honeywelrm } \\
\text { "Pressuretrolmu" meter containing } \\
\text { mercury was found on the floor. It } \\
\text { is attached to a pot that may } \\
\text { contain mercury. }\end{array}$ \\
\hline $541 \mathrm{~A}$ & & 0 & & & \\
\hline 542 & & 0 & & & $\begin{array}{l}\text { Located: } \\
\text { 1) Mercury float switch and three } \\
\text { Honeywellm pressure switches } \\
\text { with mercury bulbs in pipe } \\
\text { fitting area on shelf } \# 7 \text {. } \\
\text { 2) Located multiple Honeywelrm } \\
\text { thermostats containing mercury } \\
\text { in metal cabinet } \# D \text {. }\end{array}$ \\
\hline 543 & & 0 & & & $\begin{array}{l}\text { Honeywellim chart recorder on the } \\
\text { north wall that may contain } \\
\text { mercury. }\end{array}$ \\
\hline $543 A$ & & 1 & 0.039 & $\begin{array}{l}\text { North wall } \\
\text { floor/west end }\end{array}$ & $\begin{array}{l}\text { Several elevated readings along } \\
\text { crack where wall and floor meet. } \\
\text { A mercury spill occurred in this } \\
\text { building in the past and was } \\
\text { cleaned up. }\end{array}$ \\
\hline $543 B$ & & 0 & & & $\begin{array}{l}\text { Located: } \\
\text { 1) } 2 \text { mercury standard cells in a } \\
\text { metal cabinet on the north wall } \\
\text { of the B.G.\&U. shop. } \\
\text { 2) } 2 \text { mercury standard cells were } \\
\text { located in cabinet } \# 3 \text { on the } \\
\text { south wall of the janitor's } \\
\text { supply room. }\end{array}$ \\
\hline 544 & & 0 & & & \\
\hline 545 & & 0 & & & \\
\hline 546 & & 0 & & & \\
\hline 548 & & 0 & & & $\begin{array}{l}\text { The building consists of a } \\
\text { basement that is flooded; could not } \\
\text { access. Meters that may contain } \\
\text { mercury are visible from the hatch } \\
\text { in the roof. }\end{array}$ \\
\hline 549 & & 0 & & & \\
\hline 550 & & 0 & & & \\
\hline
\end{tabular}


Table 2.1. (continued)

\begin{tabular}{|c|c|c|c|c|c|}
\hline $\begin{array}{l}\text { Building } \\
\text { No. }\end{array}$ & $\begin{array}{l}\text { Tank } \\
\text { No. }\end{array}$ & $\begin{array}{c}\text { Detection } \\
\text { No. }\end{array}$ & $\begin{array}{c}\text { Analyzer } \\
\text { Reading, } \\
\mathrm{mg} / \mathrm{m}^{3} \mathrm{Hg}\end{array}$ & $\begin{array}{l}\text { Location of } \\
\text { Detection }\end{array}$ & Comments \\
\hline 552 & & 0 & & & \\
\hline 553 & & 0 & & & \\
\hline 555 & & 0 & & & \\
\hline 605 & & 0 & & & \\
\hline 607 & & 0 & & & \\
\hline 608 & & 0 & & & \\
\hline 611 & & 0 & & & $\begin{array}{l}\text { Large vault was locked and covid } \\
\text { not be accessed. }\end{array}$ \\
\hline 612 & & 0 & & & \\
\hline 613 & & 0 & & & \\
\hline 614 & & 0 & & & \\
\hline 515 & & 0 & & & \\
\hline 616 & & 0 & & & \\
\hline 617 & & 0 & & & \\
\hline 618 & & 0 & & & $\begin{array}{l}\text { Found a glass pressure meter } \\
\text { (CARM } 12743 \text { ) containing mercury } \\
\text { and a } 1-02 \text {. bottle of high grade } \\
\text { mercury. Both items were located } \\
\text { in row } 30 \text {, section G. }\end{array}$ \\
\hline 619 & & 0 & & & $\begin{array}{l}\text { 1) } 55 \text {-gal drum (25420), label } \\
\text { indicated mercury content. } \\
\text { The drum is located in grid } 2 \text {, } \\
\text { aisle I, row } 43 \text {. } \\
\text { 2) } 55 \text {-gal drum (40858), label } \\
\text { indicated mercury contents. } \\
\text { The drum is located in row } 32 \text {, } \\
\text { section E. }\end{array}$ \\
\hline 621 & & 0 & & & \\
\hline $621 \mathrm{~A}$ & & 0 & & & \\
\hline 622 & & 0 & & & \\
\hline 623 & & 0 & & & \\
\hline 624 & & 0 & & & \\
\hline 625 & & 0 & & & \\
\hline 627 & & 0 & & & \\
\hline $627 \mathrm{~B}$ & & 0 & & & \\
\hline 629 & & 0 & & & \\
\hline $629 \mathrm{E}$ & & 0 & & & \\
\hline 630 & & 0 & & & \\
\hline
\end{tabular}


Table 2.1. (continued)

\begin{tabular}{|c|c|c|c|c|c|}
\hline $\begin{array}{l}\text { Building } \\
\text { No. }\end{array}$ & $\begin{array}{l}\text { Tank } \\
\text { No. }\end{array}$ & $\begin{array}{l}\text { Detection } \\
\text { No. }\end{array}$ & $\begin{array}{l}\text { Analyzer } \\
\text { Reading, } \\
\mathrm{mg} / \mathrm{m}^{3} \mathrm{Hg}\end{array}$ & $\begin{array}{l}\text { Location of } \\
\text { Detection }\end{array}$ & Comments \\
\hline 631 & & 0 & & & \\
\hline $631 A$ & & 0 & & & \\
\hline 632 & & 0 & & & $\begin{array}{l}\text { There are numerous Mercoid and } \\
\text { Honeywelrm switches containing; } \\
\text { mercury on boiler system. }\end{array}$ \\
\hline 633 & & 0 & & & $\begin{array}{l}\text { Thermostat containing } 2 \text { mercury } \\
\text { bulbs on east wall. }\end{array}$ \\
\hline $633 \mathrm{~A}$ & & 0 & & & \\
\hline 633B & & 0 & & & \\
\hline 634 & & 0 & & & \\
\hline 635 & & 0 & & & \\
\hline 539 & & 0 & & & \\
\hline 643 & & 0 & & & \\
\hline $647 \mathrm{~A}$ & & 0 & & & \\
\hline $647 \mathrm{~B}$ & & 0 & & & \\
\hline $647 \mathrm{C}$ & & 0 & & & \\
\hline 647D & & 0 & & & \\
\hline 648 & & 0 & & & \\
\hline 673 & & 0 & & & \\
\hline 725 & & 0 & & & \\
\hline 726 & & 0 & & & $\begin{array}{l}\text { Could not access south side of } \\
\text { building due to bees. }\end{array}$ \\
\hline 728 & & 0 & & & \\
\hline 731 & & 0 & & & \\
\hline 732 & & 0 & & & \\
\hline 735 & & 0 & & & \\
\hline 741 & & 0 & & & $\begin{array}{l}\text { Labor building, possible past spills, } \\
\text { but no elevateu readings detected } \\
\text { during audit. }\end{array}$ \\
\hline 742 & & 1 & 0.027 & $\begin{array}{l}\text { South section, } \\
\text { north side, } \\
\text { row T }\end{array}$ & $\begin{array}{l}\text { Flow meter with a mercury pot } \\
\text { attached; no visible mercury. }\end{array}$ \\
\hline $742 \mathrm{~A}$ & & 0 & & & \\
\hline 743 & & 0 & & & $\begin{array}{l}\text { Laboratory building, possible past } \\
\text { spills; no elevated readings detected } \\
\text { during audit. A Mercoid switch } \\
\text { was detected on sump pump level } \\
\text { control system. Sump is located } \\
\text { outside on north side of building. }\end{array}$ \\
\hline
\end{tabular}


Table 2.1. (continued)

\begin{tabular}{||l|l|l|l|l|l||}
\hline $\begin{array}{c}\text { Building } \\
\text { No. }\end{array}$ & $\begin{array}{c}\text { Tank } \\
\text { No. }\end{array}$ & $\begin{array}{c}\text { Detection } \\
\text { No. }\end{array}$ & $\begin{array}{c}\text { Analyzer } \\
\text { Reading, } \\
\mathrm{mg} / \mathrm{m}^{3} \mathrm{Hg}\end{array}$ & $\begin{array}{c}\text { Location of } \\
\text { Detection }\end{array}$ & \\
\hline $743 \mathrm{~A}$ & & 0 & & & \\
\hline 744 & & 0 & & & \\
\hline 745 & & 0 & & & \\
\hline $745 \mathrm{~A}$ & 745A & 0 & & & \\
\hline $745 \mathrm{~B}$ & 745B & 0 & & & \\
\hline $745 \mathrm{C}$ & $745 \mathrm{C}$ & 0 & & & \\
\hline 748 & & 0 & & & \\
\hline 751 & & 0 & & & \\
\hline 752 & & 0 & & & \\
\hline $752 \mathrm{~A}$ & & 0 & & & \\
\hline 753 & & 0 & & & \\
\hline 754 & & 0 & & & \\
\hline 785 & & 0 & & & \\
\hline 786 & & 0 & & & \\
\hline 787 & & 0 & & & \\
\hline 788 & & 0 & & & \\
\hline 791 & & 0 & & & \\
\hline 792 & & 0 & & & \\
\hline 793 & & 0 & & & \\
\hline 794 & & 0 & & & \\
\hline 795 & & 0 & & & \\
\hline 796 & & 0 & & & \\
\hline 797 & & 0 & & & \\
\hline 798 & & 0 & & & \\
\hline 801 & & 0 & & & \\
\hline 808 & & 0 & & & \\
\hline 831 & & 0 & & & \\
\hline $831 \mathrm{~A}$ & & 0 & & & \\
\hline 833 & & 0 & & & \\
\hline 834 & & 0 & & & \\
\hline 836 & & 0 & & & \\
\hline 841 & & 0 & & & \\
\hline 846 & & 0 & & & \\
\hline 851 & & 0 & & & \\
\hline 863 & & 0 & & & \\
\hline & & & & & \\
\hline
\end{tabular}


Table 2.1. (continued)

\begin{tabular}{|c|c|c|c|c|c|}
\hline $\begin{array}{l}\text { Building } \\
\text { No. }\end{array}$ & $\begin{array}{l}\text { Tank } \\
\text { No. }\end{array}$ & $\begin{array}{l}\text { Detection } \\
\text { No. }\end{array}$ & $\begin{array}{l}\text { Analyzer } \\
\text { Reading, } \\
\mathrm{mg} / \mathrm{m}^{3} \mathrm{Hg}\end{array}$ & $\begin{array}{l}\text { Location of } \\
\text { Detection }\end{array}$ & Comments \\
\hline 864 & & 0 & & & \\
\hline 865 & & $\mathbf{0}$ & & & \\
\hline 866 & & 0 & & & \\
\hline $867 A$ & & 0 & & & \\
\hline $867 \mathrm{~B}$ & & 0 & & & \\
\hline $868 \mathrm{C}$ & & 0 & & & Building has been removed. \\
\hline 1501 & & $\mathbf{0}$ & & & $\begin{array}{l}\text { Located: } \\
\text { 1) } 2 \text { Honeywelim static pressure } \\
\text { regulators with mercury bulbs } \\
\text { on 3rd floor, south hall. } \\
\text { 2) } 2 \text { Honeywellm static pressure } \\
\text { regulators with mercury bulbs } \\
\text { on 3rd floor, north hall. } \\
\text { 3) Ashcrofte mercury switch on } \\
\text { 2nd floor, center bay. } \\
\text { 4) Manometer in lab on 2nd } \\
\text { floor, center bay. } \\
\text { 5) Honeywellm mercury switch in } \\
\text { center bay mezzanine lab, west } \\
\text { wall on overhead plumbing. } \\
\text { 6) } 2 \text { manometers, one with visible } \\
\text { mercury and one without, in } \\
\text { south mezzanine lab. } \\
\text { 7) Very old gas anatyzer in south } \\
\text { mezzanine lab that may have } \\
\text { contained mercury. } \\
\text { 8) } 11 \text { Honeywellm chart recorders } \\
\text { with } 2 \text { to } 4 \text { bulbs of mercury } \\
\text { each in the control room on } \\
\text { the northeast wall. } \\
\text { 9) } 35+\text { Panalarm meters in } \\
\text { control panel. }\end{array}$ \\
\hline $1503 \mathrm{~A}$ & & 0 & & & $\begin{array}{l}\text { Mercoid switch on a pipe next to } \\
\text { the pump. }\end{array}$ \\
\hline 1503B & & 0 & & & $\begin{array}{l}\text { Mercoid switch on a pipe next to } \\
\text { the pump. }\end{array}$ \\
\hline $1503 \mathrm{C}$ & & $\mathbf{0}$ & & & $\begin{array}{l}\text { Mercoid switch on a pipe next to } \\
\text { the pump. }\end{array}$ \\
\hline 1504 & & $\mathbf{0}$ & & & \\
\hline $1504 \mathrm{~A}$ & & 0 & & & \\
\hline $1505 \mathrm{~A}$ & & $\mathbf{0}$ & & & \\
\hline 1506 & & $\mathbf{0}$ & & & $\begin{array}{l}2 \text { Honeywelim mercury switches on } \\
\text { control panel in the northeast } \\
\text { corner of control room. }\end{array}$ \\
\hline
\end{tabular}


Table 2.1. (continued)

\begin{tabular}{|c|c|c|c|c|c|}
\hline $\begin{array}{l}\text { Building } \\
\text { No. }\end{array}$ & $\begin{array}{l}\text { Tank } \\
\text { No. }\end{array}$ & $\begin{array}{c}\text { Detection } \\
\text { No. }\end{array}$ & $\begin{array}{c}\text { Analyzer } \\
\text { Reading, } \\
\mathrm{mg} / \mathrm{m}^{3} \mathrm{Hg}\end{array}$ & $\begin{array}{l}\text { Location of } \\
\text { Detection }\end{array}$ & Comments \\
\hline 1509 & & 0 & & & \\
\hline $1510 \mathrm{~A}$ & & 0 & & & \\
\hline 1512 & & 0 & & & \\
\hline 1601 & & 0 & & - & $\begin{array}{l}\text { Located: } \\
\text { 1) } 2 \text { Pressuretrolm switches and } \\
\text { one Mercoid switch in boiler } \\
\text { room, } 2 \text { nd floor, south end. } \\
\text { 2) Honeywellm chart recorder on } \\
\text { west wall, center room of } \\
\text { south bay. } \\
\text { 3) } 2 \text { Pressuretrolm switches in } \\
\text { room B7 on panel in south } \\
\text { end. } \\
\text { 4) } 5 \text { Pressuretrolm switches from } \\
\text { north to south on east wall of } \\
\text { room B7. } \\
\text { 5) } 1 \text { Mercoid thermostat on west } \\
\text { wall of Room B7. } \\
\text { 6) } 5 \text { mercury-containing switches } \\
\text { in M-125 bomb fill machine. } \\
\text { 7) } 1 \text { mercury thermometer on } \\
\text { east side, south end of } \\
155 \text { mm shell fill machine. } \\
\text { 8) } 2 \text { Mercoid switches on west } \\
\text { side, south end of } 155 \text { mm } \\
\text { shell fill machine; also, a } \\
\text { Mercoid switch with a pot } \\
\text { attached that may contain } \\
\text { mercury. } \\
\text { 9) } 1 \text { Mercoid switch on west side, } \\
\text { north end of } 155 \text { mm shell fill } \\
\text { machine; also a Mercoid switch } \\
\text { with a pot attached that may } \\
\text { contain mercury. } \\
\text { 10) } 5 \text { mercury-containing switches } \\
\text { on west side, north end of } \\
105 \text { mm shell fill machine. } \\
\text { 11) } 2 \text { Mercoid switches on round } \\
\text { filling machines at southwest } \\
\text { end of filling room. } \\
\text { 12) Honeywellmm chart recorder on } \\
\text { west wall, south end of filling } \\
\text { room. } \\
\text { 13) } 1 \text { Panalarmm meter in storage } \\
\text { shelf with other miscellaneous } \\
\text { parts near north end of } \\
\text { building. Could not access far } \\
\text { north end of this building. }\end{array}$ \\
\hline
\end{tabular}


Table 2.1. (continued)

\begin{tabular}{|c|c|c|c|c|c|}
\hline $\begin{array}{l}\text { Building } \\
\text { No. }\end{array}$ & $\begin{array}{l}\text { Tank } \\
\text { No. }\end{array}$ & $\begin{array}{l}\text { Detection } \\
\text { No. }\end{array}$ & $\begin{array}{c}\text { Analyzer } \\
\text { Reading, } \\
\mathrm{mg} / \mathrm{m}^{3} \mathrm{Hg}\end{array}$ & $\begin{array}{l}\text { Location of } \\
\text { Detection }\end{array}$ & Comments \\
\hline $1601 \mathrm{~A}$ & & 0 & & & $\begin{array}{l}2 \text { manometers containing mercury } \\
\text { in northeast room. }\end{array}$ \\
\hline 1602 & & 0 & & & \\
\hline $1603 \mathrm{~A}$ & & 0 & & & $\begin{array}{l}\text { Mercoid switch on a pipe next to } \\
\text { the pump. }\end{array}$ \\
\hline $1603 B$ & & 0 & & & $\begin{array}{l}\text { Mercoid switch on a pipe next to } \\
\text { the pump. }\end{array}$ \\
\hline 1606 & & 0 & & & $\begin{array}{l}\text { Located: } \\
\text { 1) Thermometer on control box. } \\
\text { 2) } 2 \text { Honeywel|m chart recorders } \\
\text { with } 4 \text { mercury bulbs each in } \\
\text { assembly roorn on east wall, } \\
\text { north bay. } \\
\text { 3) } 1 \text { Honeywelrm chart recorder } \\
\text { with } 4 \text { mercury bulbs in center } \\
\text { of north bay on west wall. } \\
\text { 4) } 1 \text { chart recorder with } 8 \\
\text { mercury bulbs in assembly } \\
\text { room on west wall. } \\
\text { 5) } 3 \text { Mercoid switches located on } \\
\text { top of round filling machine in } \\
\text { the assembly rom. } \\
\text { 6) } 3 \text { mercury thermostats in } \\
\text { boiler room. } \\
\text { 7) } 1 \text { Honeywelm chart recorder } \\
\text { with } 2 \text { mercury bulbs on north } \\
\text { wall of center room of } \\
\text { assembly area. } \\
\text { 8) Numerous chart recorders that } \\
\text { may contain mercury on } \\
\text { control panel covered with } \\
\text { black plastic in control room. } \\
\text { 9) Mercoid thermostat on wall } \\
\text { opposite containment room. } \\
\text { 10) } 3 \text { thermostats containing } \\
\text { mercury in boiler room at } \\
\text { south end of building. } \\
\text { 11) } 2 \text { thermostats that may contain } \\
\text { mercury located on overhead } \\
\text { pipe in boiler room. } \\
\text { 12) } 1 \text { Honeywellm mercury- } \\
\text { containing temperature } \\
\text { recorder outside on the east } \\
\text { side of the building. }\end{array}$ \\
\hline 1607 & & 0 & & & \\
\hline
\end{tabular}


Table 2.1. (continued)

\begin{tabular}{|c|c|c|c|c|c|}
\hline $\begin{array}{l}\text { Building } \\
\text { No. }\end{array}$ & $\begin{array}{l}\text { Tank } \\
\text { No. }\end{array}$ & $\begin{array}{c}\text { Detection } \\
\text { No. }\end{array}$ & $\begin{array}{l}\text { Analyzer } \\
\text { Reading, } \\
\mathrm{mg} / \mathrm{m}^{3} \mathrm{Hg}\end{array}$ & $\begin{array}{l}\text { Location of } \\
\text { Detection }\end{array}$ & Comments \\
\hline 1611 & & 0 & & & $\begin{array}{l}\text { Located: } \\
\text { 1) } 3 \text { Honeywell }{ }^{m} \text { gas pressure } \\
\text { switches in room } 11 \text { on } \\
\text { workbench. } \\
\text { 2) } 6 \text { Mercoid switches in room } 11 \\
\text { on workbench. } \\
\text { 3) Honeywellm gas pressure } \\
\text { switches in room } 26 \text { in supply } \\
\text { crib on aisle } 800-810 \text {, shelf } E \text {. } \\
\text { 4) } 1 \text { Mercoid switch in room } 26 \\
\text { in supply crib, aisle } 800-810 \text {, } \\
\text { shelf D. } \\
\text { 5) Mercoid switch in south corner } \\
\text { of disassembly room next to } \\
\text { suit-removal room number } 2 \text {. } \\
\text { 6) Honeywellm thermostat in } 2 \text { nd } \\
\text { floor control room on south } \\
\text { wall. } \\
\text { 7) Multiple Honeywellm gas } \\
\text { pressure switches and Mercoid } \\
\text { switches in a pile behind } \\
\text { control panel on } 2 \text { nd floor. } \\
\text { 8) } 3 \text { Mercoid control switches on } \\
\text { southwest roof. }\end{array}$ \\
\hline $1611 \mathrm{~A}$ & & 0 & & & \\
\hline 1613 & & 0 & & & \\
\hline 1614 & & 0 & & & $\begin{array}{l}3 \text { Mercoid switches on top of } \\
\text { round filling machine in the } \\
\text { southeast end of the building. }\end{array}$ \\
\hline 1615 & & 0 & & & \\
\hline 1616 & & 0 & & & \\
\hline 1618 & & 0 & & & \\
\hline 1619 & & 0 & & & \\
\hline 1622 & & 0 & & & \\
\hline 1701 & & 0 & & & $\begin{array}{l}\text { Located: } \\
\text { 1) } 4 \text { Mercoid thermostats evenly } \\
\text { spaced along east wall. } \\
\text { 2) } 4 \text { Mercoid thermostats evenly } \\
\text { spaced along west wall. } \\
\text { 3) Mercoid switch on pallet in } \\
\text { row D east. } \\
\end{array}$ \\
\hline 1702 & & 0 & & & \\
\hline
\end{tabular}


Table 2.1. (continued)

\begin{tabular}{|c|c|c|c|c|c|}
\hline $\begin{array}{l}\text { Building } \\
\text { No. }\end{array}$ & $\begin{array}{l}\text { Tank } \\
\text { No. }\end{array}$ & $\begin{array}{c}\text { Detection } \\
\text { No. }\end{array}$ & $\begin{array}{l}\text { Analyzer } \\
\text { Reading, } \\
\mathrm{mg} / \mathrm{m}^{3} \mathrm{Hg}\end{array}$ & $\begin{array}{l}\text { Location of } \\
\text { Detection }\end{array}$ & Comments \\
\hline 1703 & & 0 & & & $\begin{array}{l}\text { Located: } \\
\text { 1) } 2 \text { Mercoid switches on the west } \\
\text { wall in the east bay. } \\
\text { 2) Mercoid thermostat on south } \\
\text { wall near middle door in east } \\
\text { bay. } \\
\text { 3) Mercoid switch in assembly } \\
\text { room on south side of bay. } \\
\text { As advised by TVA, did not enter } \\
\text { west end of building due to storage } \\
\text { of unknown substances. }\end{array}$ \\
\hline 1704 & & 0 & & & $\begin{array}{l}\text { Located: } \\
\text { 1) Mercoid thermostat on south } \\
\text { wall at east end next to phone } \\
\text { cabinet lighting panel L-B. } \\
\text { 2) } 1 \text { Mercoid and } 1 \text { Honeywelrm } \\
\text { thermostat on wall in } \\
\text { generator room next to lighting } \\
\text { panel L-E. } \\
\text { 3) Mercoid thermostat in } \\
\text { generator room on north wall, } \\
\text { west end. } \\
\text { 4) Mercoid thermostat in } \\
\text { compressor room on south } \\
\text { wall, west end. } \\
\text { 5) } 2 \text { Mercoid thermostats in } \\
\text { compressor room on north } \\
\text { wall. } \\
\text { 6) Honeywelpm thermostat in } \\
\text { compressor room on north } \\
\text { wall. } \\
\text { 7) } 2 \text { Mercoid thermostats in } \\
\text { boiler rom on south wall. } \\
\text { 8) } 2 \text { Mercoid thermostats in } \\
\text { boiler room on north wall. } \\
\text { 9) } 4 \text { Honeywellm Pressuretrolm } \\
\text { gauges, } 1 \text { on each of the } \\
\text { boilers facing the center aisle. }\end{array}$ \\
\hline 1705 & & 0 & & & \\
\hline 1706 & & 1 & 0.118 & $\begin{array}{l}\text { North end of } \\
\text { center wall }\end{array}$ & $\begin{array}{l}\text { Mercoid thermostat with a broken } \\
\text { mercury bulb. Traces of mercury } \\
\text { were found under the switch. }\end{array}$ \\
\hline 1707 & & 0 & & & \\
\hline
\end{tabular}


Table 2.1. (continued)

\begin{tabular}{|c|c|c|c|c|c|}
\hline $\begin{array}{l}\text { Building } \\
\text { No. }\end{array}$ & $\begin{array}{l}\text { Tank } \\
\text { No. }\end{array}$ & $\begin{array}{c}\text { Detection } \\
\text { No. }\end{array}$ & $\begin{array}{c}\text { Analyzer } \\
\text { Reading, } \\
\mathrm{mg} / \mathrm{m}^{3} \mathrm{Hg}\end{array}$ & $\begin{array}{l}\text { Location of } \\
\text { Detection }\end{array}$ & Comments \\
\hline 1710 & & $\mathbf{0}$ & & & $\begin{array}{l}\text { Located: } \\
\text { 1) } 3 \text { bottles containing mercury- } \\
\text { contaminated water in room } \\
11 . \\
\text { 2) Honeywell } \\
\text { room thermostat in } 36 \text { on north wall. }\end{array}$ \\
\hline 1711 & & 0 & & & \\
\hline 1712 & & $\mathbf{0}$ & & & $\begin{array}{l}\text { Located: } \\
\text { 1) } 2 \text { Honeywellm Pressuretrolm } \\
\text { gauges, } 1 \text { on northeast side of } \\
\text { east furnace and } 1 \text { on north- } \\
\text { east side of west furnace. } \\
\text { 2) } 2 \text { Honeywellm Pressuretrolm } \\
\text { gauges, } 1 \text { on west side of east } \\
\text { furnace and } 1 \text { on west side of } \\
\text { west furnace. }\end{array}$ \\
\hline 1713 & & $\mathbf{0}$ & & & \\
\hline 1717 & & 0 & & & \\
\hline 1718 & & 0 & & & \\
\hline 1719 & & 0 & & & \\
\hline 1727 & & $\mathbf{0}$ & & & \\
\hline 1730 & & 0 & & & Inaccessible due to bees. \\
\hline 1734 & & 0 & & & \\
\hline 1735 & & 0 & & & \\
\hline NN0104 & & $\mathbf{0}$ & & & $\begin{array}{l}\text { Broken temperature gauge that } \\
\text { may contain mercury (no visible } \\
\text { mercury). }\end{array}$ \\
\hline NN0105 & & $\mathbf{0}$ & & & \\
\hline NN0106 & & 0 & & & \\
\hline NN0111 & & 0 & & & \\
\hline NN0112 & & 0 & & & \\
\hline NN0115 & & 0 & & & \\
\hline NN0116 & & 0 & & & \\
\hline NN0601 & & 0 & & & Building no longer exists. \\
\hline NN0602 & & 0 & & & \\
\hline NNO603 & & 0 & & & \\
\hline NN0903 & & 0 & & & Access not controlled by PMRMA \\
\hline NN1201 & & 0 & & & Building has been removed. \\
\hline NN1213 & & $\mathbf{0}$ & & & Building has been removed. \\
\hline
\end{tabular}


Table 2.1. (continued)

\begin{tabular}{|c|c|c|c|c|c|}
\hline $\begin{array}{l}\text { Building } \\
\text { No. }\end{array}$ & $\begin{array}{l}\text { Tank } \\
\text { No. }\end{array}$ & $\begin{array}{l}\text { Detection } \\
\text { No. }\end{array}$ & $\begin{array}{c}\text { Analyzer } \\
\text { Reading, } \\
\mathrm{mg} / \mathrm{m}^{3} \mathrm{Hg}\end{array}$ & $\begin{array}{l}\text { Location of } \\
\text { Detection }\end{array}$ & Comments \\
\hline $\mathrm{NN} 2301$ & & 0 & & & \\
\hline NN2403 & & 0 & & & \\
\hline NN2502 & & 0 & & & \\
\hline $\mathrm{NN} 2503$ & & $\mathbf{0}$ & & & \\
\hline T0026 & T0026 & 0 & & & \\
\hline T0064 & T0064 & 0 & & & \\
\hline $\mathrm{T} 1040$ & $\mathrm{~T} 1040$ & 0 & & & Temperature probe $\left(500^{\circ} \mathrm{F}\right)$. \\
\hline T1606 & T1606 & 0 & & & Tank could not be located. \\
\hline TF0101 & T1219 & 0 & & & \\
\hline TF0101 & $\mathrm{T} 1220$ & 0 & & & \\
\hline TF0101 & $\mathrm{T} 1267$ & 0 & & & \\
\hline TF0101 & $\mathrm{T} 1296$ & 0 & & & \\
\hline TF0101 & $\mathrm{T} 1307$ & 0 & & & \\
\hline TF0101 & T1394 & $\mathbf{0}$ & & & $\begin{array}{l}\text { High temperature thermometer on } \\
\text { south side of tank. }\end{array}$ \\
\hline TF0102 & T1010 & $\mathbf{0}$ & & & \\
\hline TF0102 & $\mathrm{T} 1216$ & 0 & & & \\
\hline TF0102 & $\mathrm{T} 1340$ & 0 & & & Tank had been removed. \\
\hline TF0102 & $\mathrm{V} 1230$ & 0 & & & Tank had been removed. \\
\hline TF0102A & T0014 & 0 & & & \\
\hline TF0102A & $\mathrm{T} 0015$ & 0 & & & \\
\hline TF0102A & T0160 & 0 & & & \\
\hline TF0102A & T0161 & 0 & & & \\
\hline TF0102A & T0164 & 0 & & & \\
\hline TF0102A & T0165 & $\mathbf{0}$ & & & \\
\hline TF0103 & T0065 & 0 & & & $\begin{array}{l}\text { Temperature controller }\left(250^{\circ} \mathrm{F}\right) \text {, } \\
\text { west side of tank. }\end{array}$ \\
\hline TF0103 & T0066 & 0 & & & Tank has been removed. \\
\hline TF0103 & T0075 & 0 & & & Temperature gauge $\left(<160^{\circ} \mathrm{F}\right)$. \\
\hline TF0103 & T0076 & 0 & & & \\
\hline TF0103 & T0077 & $\mathbf{0}$ & & & \\
\hline TF0103 & T0078 & 0 & & & \\
\hline TF0103 & T0079 & 0 & & & \\
\hline TF0103 & To080 & 0 & & & \\
\hline TF0103 & T0081 & $\mathbf{0}$ & & & \\
\hline
\end{tabular}


Table 2.1. (continued)

\begin{tabular}{|c|c|c|c|c|c|}
\hline $\begin{array}{l}\text { Building } \\
\text { No. }\end{array}$ & $\begin{array}{l}\text { Tank } \\
\text { No. }\end{array}$ & \begin{tabular}{|c|} 
Detection \\
No. \\
\end{tabular} & $\begin{array}{c}\text { Analyzer } \\
\text { Reading, } \\
\mathrm{mg} / \mathrm{m}^{3} \mathrm{Hg}\end{array}$ & $\begin{array}{l}\text { Location of } \\
\text { Detection }\end{array}$ & Comments \\
\hline TF0103 & T0082 & 0 & & & \\
\hline TF0103 & T0178 & 0 & & & $\begin{array}{l}\text { Instrumentation on catwalk not } \\
\text { checked. }\end{array}$ \\
\hline TF0103 & T0197 & 0 & & & $\begin{array}{l}\text { Instrumentation on catwalk not } \\
\text { checked. }\end{array}$ \\
\hline TF0103 & T1124 & $\mathbf{0}$ & & & $\begin{array}{l}\text { Instrumentation on east and west } \\
\text { side of tank. }\end{array}$ \\
\hline TF0103 & $\mathrm{T} 1202$ & 0 & & & \\
\hline TF0103 & $\mathrm{T} 1203$ & 0 & & & \\
\hline TF0103 & $\mathrm{T} 1204$ & 0 & & & $\begin{array}{l}\text { Thermostat on south end of tank } \\
\left(300^{\circ} \mathrm{F}\right) \text {. }\end{array}$ \\
\hline TF0103 & T1272 & 0 & & & \\
\hline TF0103 & T1273 & 0 & & & \\
\hline TF0103 & T1324 & 0 & & & \\
\hline TF0103 & T1377 & $\mathbf{0}$ & & & \\
\hline TF0103 & T1446 & 0 & & & $\begin{array}{l}\text { Recorder on west side of tank, } \\
\text { instrumentation above. }\end{array}$ \\
\hline TF0103 & V1147 & 0 & & & \\
\hline TF0104 & T0019 & 0 & & & $\begin{array}{l}\text { High temperature probe on south } \\
\text { end of tank. }\end{array}$ \\
\hline TF0104 & T1128 & 0 & & & Tank has been removed. \\
\hline TF0104 & T1129 & 0 & & & Tank has been removed \\
\hline TF0104 & T1132 & 0 & & & Tank has been removed. \\
\hline TF0104 & $\mathrm{T} 1133$ & 0 & & & Tank has been removed. \\
\hline TF0104 & $\mathrm{T} 1135$ & 0 & & & \\
\hline TF0104 & T1139 & 0 & & & \\
\hline TF0104 & $\mathrm{T} 1140$ & 0 & & & Tank has been removed. \\
\hline TF0104 & $\mathrm{T} 1148$ & $\mathbf{0}$ & & & $\begin{array}{l}\text { Possible mercury-containing } \\
\text { instrument on top of tank. High } \\
\text { temperature thermometer and flow } \\
\text { meter on sides of tank. }\end{array}$ \\
\hline TF0104 & $\mathrm{T} 1149$ & $\mathbf{0}$ & & & $\begin{array}{l}\text { Possible mercury-containing } \\
\text { instrument on top of tank. High } \\
\text { temperature thermometer and flow } \\
\text { meter on sides of tank. }\end{array}$ \\
\hline TF0104 & $\mathrm{T} 1150$ & 0 & & & \\
\hline TF0104 & $\mathrm{T} 1151$ & 0 & & & \\
\hline TF0104 & T1222 & 0 & & & \\
\hline
\end{tabular}


Table 2.1. (continued)

\begin{tabular}{|c|c|c|c|c|c|}
\hline $\begin{array}{l}\text { Building } \\
\text { No. }\end{array}$ & $\begin{array}{l}\text { Tank } \\
\text { No. }\end{array}$ & $\begin{array}{c}\text { Detection } \\
\text { No. }\end{array}$ & $\begin{array}{c}\text { Analyzer } \\
\text { Reading, } \\
\mathrm{mg} / \mathrm{m}^{3} \mathrm{Hg}\end{array}$ & $\begin{array}{l}\text { Location of } \\
\text { Detection }\end{array}$ & Comments \\
\hline TF0104 & T1246 & 0 & & & $\begin{array}{l}\text { Possible mercury-containing } \\
\text { instrument on top of tank. High } \\
\text { temperature thermometer and flow } \\
\text { meter on sides of tank. }\end{array}$ \\
\hline TF0104 & T1247 & 0 & & & $\begin{array}{l}\text { Possible mercury-containing } \\
\text { instrument on top of tank. High } \\
\text { temperature thermometer and flow } \\
\text { meter on sides of tank. }\end{array}$ \\
\hline TF0104 & T1322 & 0 & & & \\
\hline TF0104 & T1323 & 0 & & & \\
\hline TF0104 & T1463 & 0 & & & \\
\hline TF0104 & V1250 & 0 & & & \\
\hline TF0104 & V1254A & 0 & & & \\
\hline TF0104 & V1254B & 0 & & & \\
\hline TF0105 & V1500 & 0 & & & \\
\hline TF0105 & T1501 & 0 & & & \\
\hline TF0105 & $\mathrm{T} 1502$ & 0 & & & \\
\hline TF0105 & T1503 & 0 & & & \\
\hline TFO105 & T1504 & 0 & & & $\begin{array}{l}\text { Several instruments and a 1-gal pot } \\
\text { that may contain mercury are on } \\
\text { top of tank. }\end{array}$ \\
\hline TF0105 & T1505 & 0 & & & \\
\hline TF0105 & T1506 & 0 & & & \\
\hline TF0105 & T1507 & 0 & & & \\
\hline TF0105 & T1508 & 0 & & & \\
\hline TF0105 & T1509 & 0 & & & \\
\hline TF0105 & $\mathrm{T} 1510$ & 0 & & & \\
\hline TF0105 & $\mathrm{T} 1511$ & 0 & & & $\begin{array}{l}\text { High temperature probes on east } \\
\text { and west sides of tank. }\end{array}$ \\
\hline TF0105 & $\mathrm{T} 1512$ & 0 & & & \\
\hline TF0105 & T1513 & 0 & & & \\
\hline TF0105 & T1514 & 0 & & & \\
\hline TF0105 & T1515 & 0 & & & \\
\hline TF0105 & \begin{tabular}{|l|} 
T1516 \\
\end{tabular} & 0 & & & \\
\hline TF0105 & T1566 & 0 & & & \\
\hline TF0105 & T1569 & 0 & & & \\
\hline TF0105 & T1570 & 0 & & & \\
\hline
\end{tabular}


Table 2.1. (continued)

\begin{tabular}{|c|c|c|c|c|c|}
\hline $\begin{array}{l}\text { Building } \\
\text { No. }\end{array}$ & $\begin{array}{l}\text { Tank } \\
\text { No. }\end{array}$ & $\begin{array}{l}\text { Detection } \\
\text { No. }\end{array}$ & $\begin{array}{l}\text { Analyzer } \\
\text { Reading, } \\
\mathrm{mg} / \mathrm{m}^{3} \mathrm{Hg}\end{array}$ & $\begin{array}{l}\text { Location of } \\
\text { Detection }\end{array}$ & Comments \\
\hline TF0105A & V1255 & 0 & & & \\
\hline TF0105A & V1259 & 0 & & & \\
\hline TF0105A & V1264 & 0 & & & Tank has been removed. \\
\hline TF0105A & V1265 & 0 & & & Tank has been removed. \\
\hline TF0105A & V1267 & 0 & & & Could not locate tank. \\
\hline TF0105A & V1270 & 0 & & & \\
\hline TF0105A & $V 1313$ & 0 & & & \\
\hline TF0106 & T0058 & 0 & & & \\
\hline TF0106 & T02S7 & 0 & & & \\
\hline TF0106 & T1027 & 0 & & & \\
\hline TF0106 & $\mathrm{T} 1315$ & 0 & & & \\
\hline TF0106 & V1156 & 0 & & & \\
\hline TF0106 & V1187 & 0 & & & \\
\hline TF0107 & T0026 & 0 & & & \\
\hline TF0107 & T0027 & 0 & & & \\
\hline Tro107 & T0139 & 0 & & & \\
\hline TF0107 & T0190 & 0 & & & \\
\hline TF0108 & T1235 & 0 & & & \\
\hline TF0108 & $\mathrm{T} 1253$ & 0 & & & $\begin{array}{l}\text { Temperature probe }\left(300^{\circ} \mathrm{F}\right) \text {, on } \\
\text { south end of tank. }\end{array}$ \\
\hline TF0108 & T1279 & 0 & & & \\
\hline TF0108 & $\mathrm{T} 1288$ & 0 & & & \\
\hline TF0108 & T1289 & 0 & & & \\
\hline TF0108 & $\mathrm{T} 1290$ & 0 & & & \\
\hline TF0108 & $\mathrm{T} 1291$ & 0 & & & \\
\hline 'ГF0108 & $\mathrm{T} 1305$ & 0 & & & \\
\hline TF0108 & $\mathrm{T} 1433$ & 0 & & & \\
\hline TF0108 & V1186 & 0 & & & \\
\hline TF0108 & V1253 & 0 & & & $\begin{array}{l}\text { Possible mercury-containing } \\
\text { instrumentation. }\end{array}$ \\
\hline TF0108 & T1307 & 0 & & & \\
\hline TF0110 & T1127 & 0 & & & \\
\hline TF0110 & $\mathrm{T} 1134$ & 0 & & & \\
\hline TF0110 & T1146 & 0 & & & $\begin{array}{l}\text { Could not access tank; fenced off } \\
\text { by Shell. }\end{array}$ \\
\hline
\end{tabular}


Table 2.1. (continued)

\begin{tabular}{||c|c|c|c|c|c|}
\hline $\begin{array}{c}\text { Building } \\
\text { No. }\end{array}$ & $\begin{array}{c}\text { Tank } \\
\text { No. }\end{array}$ & $\begin{array}{c}\text { Detection } \\
\text { No. }\end{array}$ & $\begin{array}{c}\text { Analyzer } \\
\text { Reading, } \\
\text { mg/m } \mathrm{m}^{3} \mathrm{Hg}\end{array}$ & $\begin{array}{c}\text { Location of } \\
\text { Detection }\end{array}$ & \\
\hline TF0110 & T1147 & 0 & & & $\begin{array}{c}\text { Could not access tank; fenced off } \\
\text { by Shell. }\end{array}$ \\
\hline TF0110 & T1171 & 0 & & & \\
\hline TF0110 & T1173 & 0 & & & \\
\hline TF0110 & T1215 & 0 & & & \\
\hline TF0512 & V1001 & 0 & & & \\
\hline TF0512 & V1002 & 0 & & & \\
\hline TF0628 & T0628A & 0 & & & \\
\hline TF0628 & T0629A & 0 & & & \\
\hline TF0628 & T0629B & 0 & & & \\
\hline TF0628 & T0629C & 0 & & & \\
\hline TF0628 & T0629D & 0 & & & \\
\hline TF0628 & T0648A & 0 & & & \\
\hline TF0628 & T0648B & 0 & & & \\
\hline TF1402 & T41104 & 0 & & & \\
\hline TF1402 & T41115 & 0 & & & \\
\hline TF1402 & T49368 & 0 & & & \\
\hline TF1402 & T49369 & 0 & & & \\
\hline TF1402 & T49370 & 0 & & & \\
\hline TF1402 & T49371 & 0 & & & \\
\hline TF1402 & T49372 & 0 & & & \\
\hline TF1402 & T49373 & 0 & & & \\
\hline TF1403 & T0001 & 0 & & & \\
\hline TF1403 & T0002 & 0 & & & \\
\hline TF1403 & T0003 & 0 & & & \\
\hline TF1403 & T0004 & 0 & & & \\
\hline TF1403 & T0005 & 0 & & & \\
\hline TF1404 & 1404 & 0 & & & \\
\hline TF1405 & T0001 & 0 & & & \\
\hline TF1405 & T0002 & 0 & & & \\
\hline TF1502 & F5109 & 0 & & & \\
\hline TF1502 & F5110 & 0 & & & \\
\hline TF1502 & F5111 & 0 & & & \\
\hline TF1502 & F5112 & 0 & & & \\
\hline TF1502 & F5601 & 0 & & & \\
\hline
\end{tabular}


Table 2.1. (continued)

\begin{tabular}{|l|l|l|l|l|l|}
\hline $\begin{array}{c}\text { Building } \\
\text { No. }\end{array}$ & $\begin{array}{c}\text { Tank } \\
\text { No. }\end{array}$ & $\begin{array}{c}\text { Detection } \\
\text { No. }\end{array}$ & $\begin{array}{c}\text { Analyzer } \\
\text { Reading, } \\
\mathrm{mg} / \mathrm{m}^{3} \mathrm{Hg}\end{array}$ & $\begin{array}{c}\text { Location of } \\
\text { Detection }\end{array}$ & \\
\hline TF1502 & F5602 & 0 & & & Comments \\
\hline TF1505 & F05102 & 0 & & & \\
\hline TF1505 & F05103 & 0 & & & \\
\hline TF1505 & F05104 & 0 & & & \\
\hline TF1505 & F05105 & 0 & & & \\
\hline TF1505 & F05106 & 0 & & & \\
\hline TF1505 & F05107 & 0 & & & \\
\hline TF1505 & F05108 & 0 & & & \\
\hline TF1505 & F50097 & 0 & & & \\
\hline TF1505 & F50098 & 0 & & & \\
\hline TF1505 & F50099 & 0 & & & \\
\hline TF1508 & T0001 & 0 & & & \\
\hline TF2501 & F40007 & 0 & & & \\
\hline TF2501 & F50117 & 0 & & & \\
\hline TF2501 & F50120 & 0 & & & \\
\hline TF2501 & F50123 & 0 & & & \\
\hline TF2501 & F50393 & 0 & & & \\
\hline TF2501 & F50394 & 0 & & & \\
\hline TF2501 & F50670 & 0 & & & \\
\hline TF2501 & F50967 & 0 & & & \\
\hline TF2501 & G50830 & 0 & & & \\
\hline TF2501 & G50831 & 0 & & & \\
\hline & & & & & \\
\hline
\end{tabular}


Table 2.2. Tanks and buildings not audited

\begin{tabular}{lll}
\hline & \multicolumn{2}{c}{ TANKS } \\
\hline Tank Farm & Tank number & Reason for not surveying \\
\hline TF0102 & V1230 & Tank has been removed \\
TF0102 & T1340 & Tank has been removed \\
TF0103 & T0066 & Tank has been removed \\
TF0104 & T1128 & Tank has been removed \\
TF0104 & T1129 & Tank has been removed \\
TF0104 & T1132 & Tank has been removed \\
TF0104 & T1133 & Tank has been removed \\
TF0104 & T1140 & Tank has been removed \\
TF0105A & V1264 & Tank has been removed \\
TF0105A & V1265 & Tank has been removed \\
TF0105A & V1267 & Could not locate tank \\
T1606 & T1606 & T1146 \\
TF0110 & T1147 & Could not locate tank \\
TF0110 & & Could not access, fenced off by Shell \\
\hline
\end{tabular}


Table 2.2 (continued)

\begin{tabular}{lll}
\hline & \multicolumn{2}{c}{ BULDINGS } \\
\hline Building No. & \multicolumn{1}{c}{ Description } & Reason for not surveying \\
\hline 344 & $\begin{array}{l}\text { 3 liquid chlorine tank } \\
\text { saddles }\end{array}$ & Building has been removed \\
& $\begin{array}{l}\text { Flammable material } \\
\text { storehouse }\end{array}$ & Boarded up, no access \\
$383 \mathrm{~A}$ & Officer's Club storage & Building has been removed \\
548 & $\begin{array}{l}\text { Process water pumping } \\
\text { station (underground) }\end{array}$ & Flooded \\
NN0601 & $\begin{array}{l}\text { Loading dock } \\
\text { Recreation building }\end{array}$ & Building has been removed \\
$868 \mathrm{C}$ & $\begin{array}{l}\text { Office/ton container } \\
\text { storage shed }\end{array}$ & $\begin{array}{l}\text { Building has been removed } \\
\text { VORTAC station }\end{array}$ \\
NN0903 & $\begin{array}{l}\text { Locked, not under PMRMA } \\
\text { control }\end{array}$ \\
NN1201 & Long metal shed & Building has been removed \\
NN1213 & Maintenance shop & Building has been removed \\
Sentry station/gate house & Inaccessible due to bees \\
\hline
\end{tabular}


In addition, the team was unable to access portions of some buildings. The locations and reasons for partial surveys are listed in the comments section of Table 2.1.

\section{DETECTIONS}

Detections during the audit are defined as above-zero readings on the Jerome 431- $\mathrm{X}^{\mathrm{m}}$ Mercury Vapor Analyzer. The twelve detections located are presented in Table 2.3. Visible mercury spills were the most critical of these, with the remaining detections attributed to mercury-containing instrumentation and residual mercury from past spills. There were no visible mercury spills in these remaining detections. Detections in five buildings $(241,321,346,411 \mathrm{~A}$, and 1706) exceed the Occupational Safety and Health Administration's (OSHA) permissible exposure limit (PEL) of $0.100 \mathrm{mg} / \mathrm{m}^{3}$ for mercury.

\subsubsection{Visible Mercury Spills}

Visible mercury spills were found in Buildings 321, 346, 411a, and 1706. The spills in Buildings 321,346, and 411a were from instruments with leaking mercury pots. Figure 1 top shows a typical instrument control panel utilizing mercury pots; Fig. 1 bottom shows a back view of a portion of the same instrument panel with the mercury pot attached to the back of a round chart recorder. The spill detected in Building 1706 was the result of a broken thermostat. In addition to these four mercury spills, there were two buckets in Building 346 containing bottles of mercury drained from old mercury pots. Mercury vapors were leaking from these containers. 
Table 23. Mercury detections

\begin{tabular}{|c|c|c|c|c|c|}
\hline $\begin{array}{l}\text { Building } \\
\text { No. }\end{array}$ & $\begin{array}{l}\text { Tank } \\
\text { No. }\end{array}$ & $\begin{array}{l}\text { Detection } \\
\text { No. }\end{array}$ & $\begin{array}{l}\text { Analyzer } \\
\text { Reading, } \\
\mathrm{mg} / \mathrm{m}^{3} \mathrm{Hg}\end{array}$ & $\begin{array}{l}\text { Location of } \\
\text { Detection }\end{array}$ & Comments \\
\hline 241 & & 1 & $0.128^{a}$ & $\begin{array}{l}\text { Old laboratory } \\
\text { room }\end{array}$ & $\begin{array}{l}\text { Elevated readings inside the } \\
\text { center lab bench and the } \\
\text { northern-most bottom cabinet, } \\
\text { west side. The cabinet is marked } \\
\text { asbestos (no visible mercury). }\end{array}$ \\
\hline 243 & & 1 & 0.010 & $\begin{array}{l}\text { East wall/ } \\
\text { switchroom/ } \\
\text { 2nd floor }\end{array}$ & $\begin{array}{l}\text { Elevated readings in the switch- } \\
\text { room ( } 2 \text { nd room from west end) } \\
\text { on an in-line pressure gauge } \\
\text { (manometer) containing mercury. } \\
\text { A pot that may contain mercury } \\
\text { was also found. }\end{array}$ \\
\hline 321 & & 1 & $0.764^{a}$ & $\begin{array}{l}\text { North wally } \\
\text { 2nd floor }\end{array}$ & $\begin{array}{l}\text { 1) Behind an instrument panel, } \\
\text { visible spills of mercury were } \\
\text { detected on the floor. Most } \\
\text { instruments have pots } \\
\text { containing mercury. These } \\
\text { instruments are still in use. } \\
\text { 2) Throughout the building are } \\
\text { Mercoid switches containing } \\
\text { small amounts of mercury. } \\
\text { These switches are still in use. }\end{array}$ \\
\hline 325 & & 1 & 0.040 & $\begin{array}{l}\text { North wall } \\
\text { 1st floor }\end{array}$ & $\begin{array}{l}\text { Elevated readings of mercury } \\
\text { were detected on the floor behind } \\
\text { the control panel. Instruments in } \\
\text { the panel have pots containing } \\
\text { mercury. }\end{array}$ \\
\hline 325 & & 2 & 0.006 & $\begin{array}{l}\text { 2nd floor/ } \\
\text { southeast } \\
\text { corner }\end{array}$ & $\begin{array}{l}\text { The instrument assembly room } \\
\text { was used to drain mercury from } \\
\text { old meters. Slightly elevated } \\
\text { readings were detected in the } \\
\text { corners of the room. }\end{array}$ \\
\hline 346 & & 1 & $0.860^{\circ}$ & Southeast wall & $\begin{array}{l}\text { A mercury spill was found in the } \\
\text { meter storage area. Small pools } \\
\text { of mercury are visible below an } \\
\text { old instrument. Additional } \\
\text { mercury-containing meters are } \\
\text { stored in this area. }\end{array}$ \\
\hline 346 & & 2 & $0.342^{\circ}$ & $\begin{array}{l}\text { North wall } \\
\text { westernmost } \\
\text { door }\end{array}$ & $\begin{array}{l}\text { Two 5-gal buckets containing } \\
\text { approximately } 3 \text { gal of mercury in } \\
\text { separate plastic containers were } \\
\text { found near the northwest door. }\end{array}$ \\
\hline $411 A$ & & 1 & $0.480^{\circ}$ & $\begin{array}{l}\text { Northeast } \\
\text { corner under } \\
\text { instrument }\end{array}$ & $\begin{array}{l}\text { Visible mercury spill from a } \\
\text { mercury pot in a recorder. }\end{array}$ \\
\hline
\end{tabular}


Table 23. (continued)

\begin{tabular}{||l|c|c|c|l|l||}
\hline $\begin{array}{c}\text { Building } \\
\text { No. }\end{array}$ & $\begin{array}{c}\text { Tank } \\
\text { No. }\end{array}$ & $\begin{array}{c}\text { Detection } \\
\text { No. }\end{array}$ & $\begin{array}{c}\text { Analyzer } \\
\text { Reading, } \\
\mathrm{mg}^{3} \mathrm{mg}\end{array}$ & $\begin{array}{l}\text { Location of } \\
\text { Detection }\end{array}$ & \multicolumn{1}{|c|}{ Comments } \\
\hline 433 & 1 & 0.024 & $\begin{array}{l}\text { South } \\
\text { corridor/south } \\
\text { side of bldg. }\end{array}$ & $\begin{array}{l}2 \text { chart recorders in south } \\
\text { corridor, soutb side of building. } \\
\text { No visible mercury. }\end{array}$ \\
\hline $543 \mathrm{~A}$ & & 1 & 0.039 & $\begin{array}{l}\text { North wall/ } \\
\text { floor/west end }\end{array}$ & $\begin{array}{l}\text { Several elevated readings along } \\
\text { crack where wall and floor meet. } \\
\text { A mercury spill occurred in this } \\
\text { building in the past and was } \\
\text { cleaned up. }\end{array}$ \\
\hline 742 & 1 & 0.027 & $\begin{array}{l}\text { South section/ } \\
\text { north side/ } \\
\text { row T }\end{array}$ & $\begin{array}{l}\text { Fow meter with a mercury pot } \\
\text { attached; no visible mercury. }\end{array}$ \\
\hline 1706 & 1 & $0.118^{\circ}$ & $\begin{array}{l}\text { North end of } \\
\text { center wall }\end{array}$ & $\begin{array}{l}\text { Mercoid thermostat with a broken } \\
\text { mercury bulb. Traces uf mercury } \\
\text { were found under the switch. }\end{array}$ \\
\hline
\end{tabular}

- Readings exceed the OSHA PEL of $0.100 \mathrm{mg} / \mathrm{m}^{3}$ for an 8-hour period. 


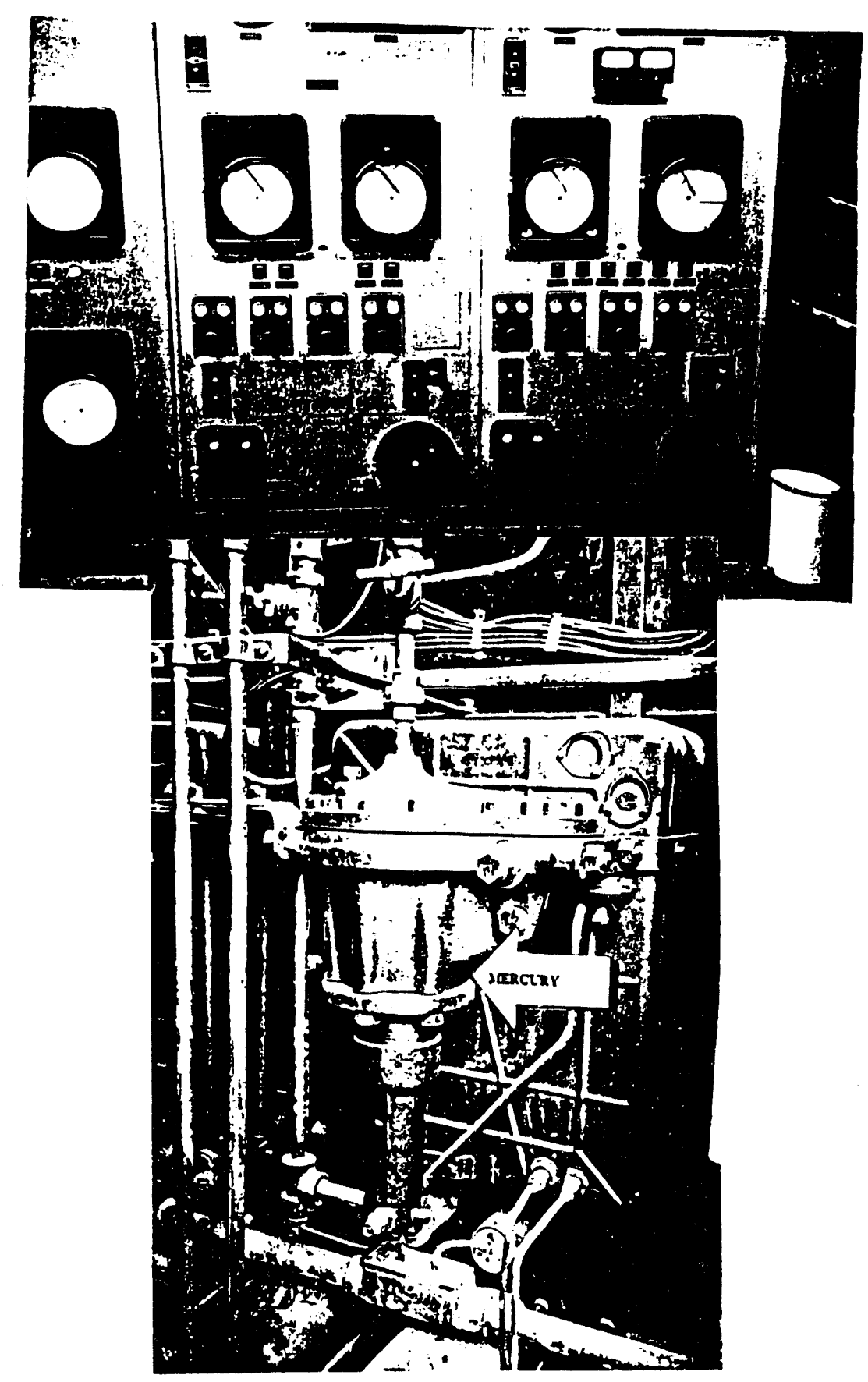

Fig. 1. Front view of instrument control panel and rear view showing mercury pot. 


\section{Other Detections}

Buildings 325 and 742 each had mercury pots emitting detectable mercury vapors. Neither of these buildings had visible mercury spills.

Reported past mercury spills were closely investigated, and three of these areas in Buildings 241, 325, and 543A showed detectable mercury vapor with no visible mercury. The detection location in Building $\mathbf{3 2 5}$ was an area used to drain old mercury pots.

Building 433 contains two chart recorders. Mercury vapor was detected inside the recorders through a small hole; however, since the doors of both recorders were rusted shut, the presence of mercury bulbs could not be confirmed.

The remaining detection was at an old manometer located in Building 243. A similar mercury-containing manometer is shown in Fig. 2.

\section{INSTRUMENTATION}

In addition to the mercury-containing instruments shown in Figs. 1 and 2 , several other commonly found instruments containing mercury bulbs were photographed to aid with any remedial action that may follow. Figure 3 shows two Mercoid controls and a Mercoid thermostat, both commonly found on machinery at the RMA. The color of the controls varies from black to green. All are round, with mercury bulbs visible through a glass window. A Mercoid thermostat was found in Building 1706 with a broken mercury bulb. Another common mercurycontaining thermostat is the round Honeywell ${ }^{m}$ thermostat (no picture available). Not all of the thermostats used at RMA contain mercury.

Figure 4 shows the front and back of a Honeywellm chart recorder and the locations of the mercury bulbs. These recorders typically had two to four mercury bulbs. 

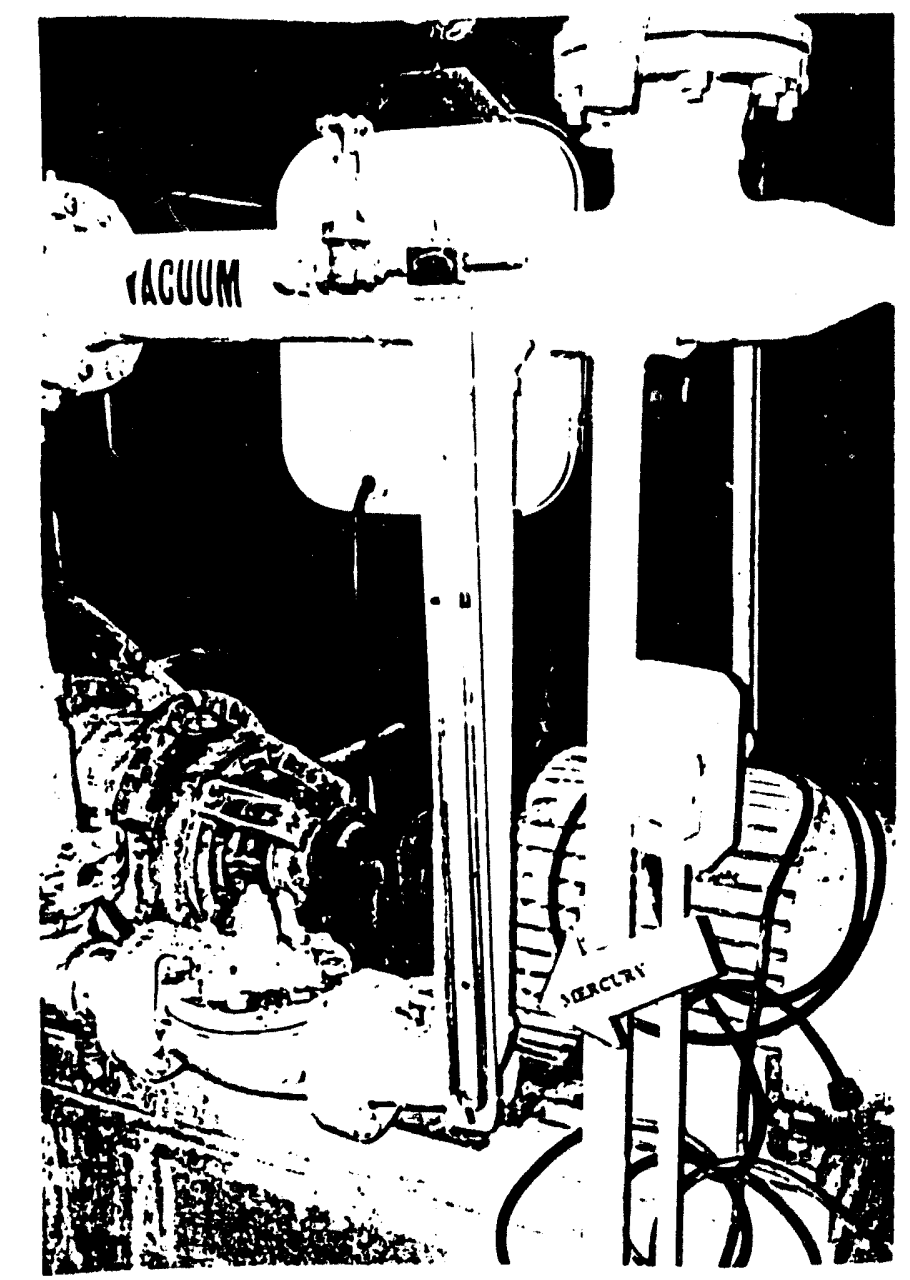

Fig. 2. Mercury-containing manometer. 


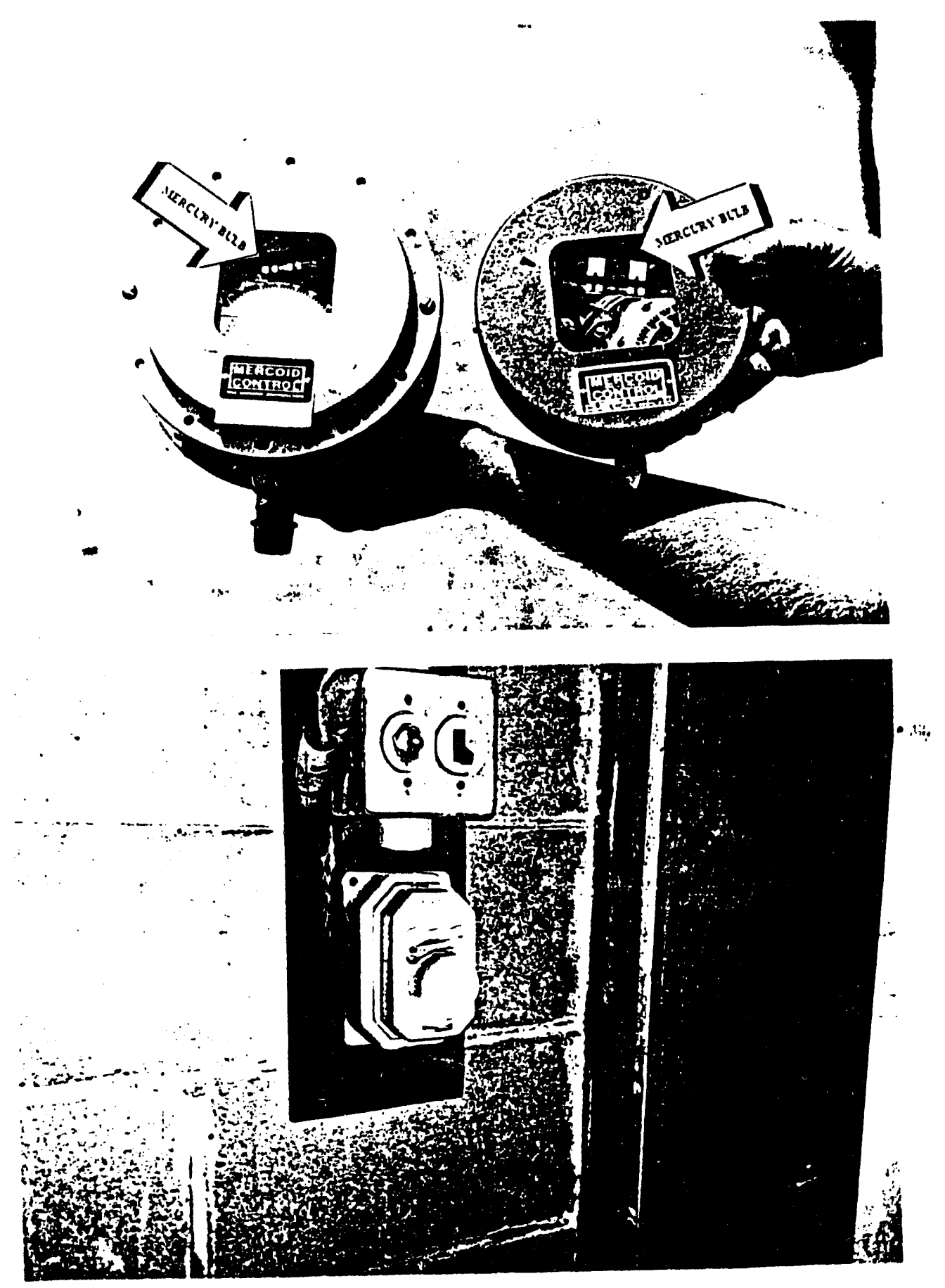

Fig. 3. Mercoid controls and Mercoid thermostat. 


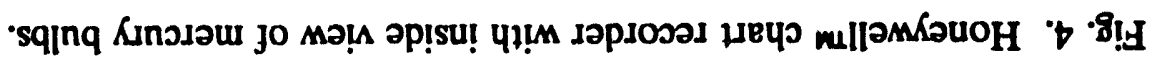
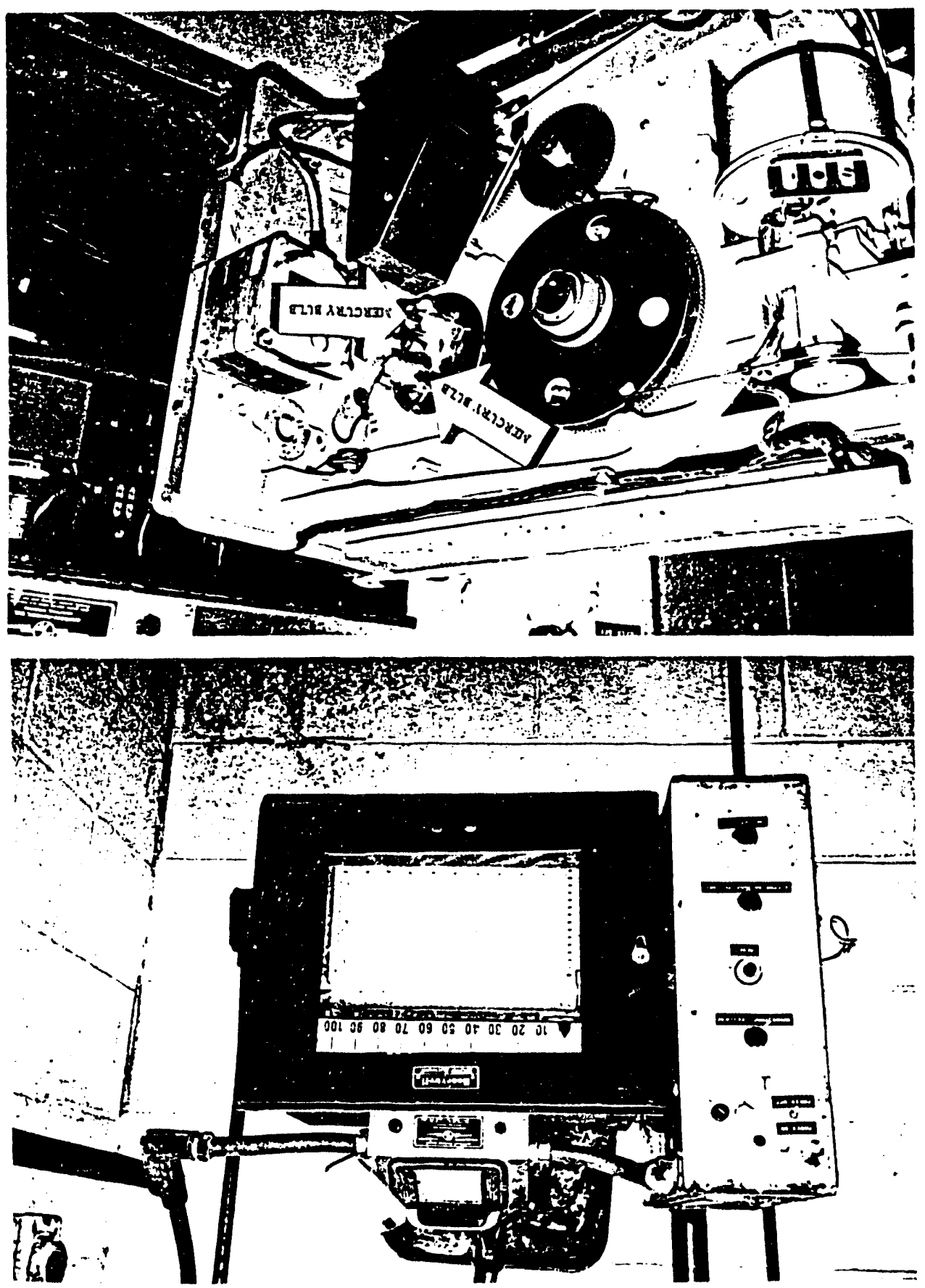
Figure 5 shows a control panel with 10 Panalarm ${ }^{m}$ controls. Also shown are an individual Panalarm ${ }^{m}$ detached from the control panel and a side view of the instrument.

Figure 6 and the top of Fig. 7 are pictures of Pressuretrolm instruments. Several different types of these instruments were located at the RMA, some appearing to be newer than others. Mercury bulbs are indicated.

A Honeywellim gas pressure switch appears as the bottom picture in Fig. 7. Many of these switches are located in boxes in storage areas and supply cabinets; however, none were located on machinery.

In addition to those shown in the figures, many other instruments were found and are listed in Table 2.1 with brief descriptions of locations. It should be noted that instruments suspected of containing mercury are also on this list. The placement or condition of the instrument prevented confirmation of mercury content. 


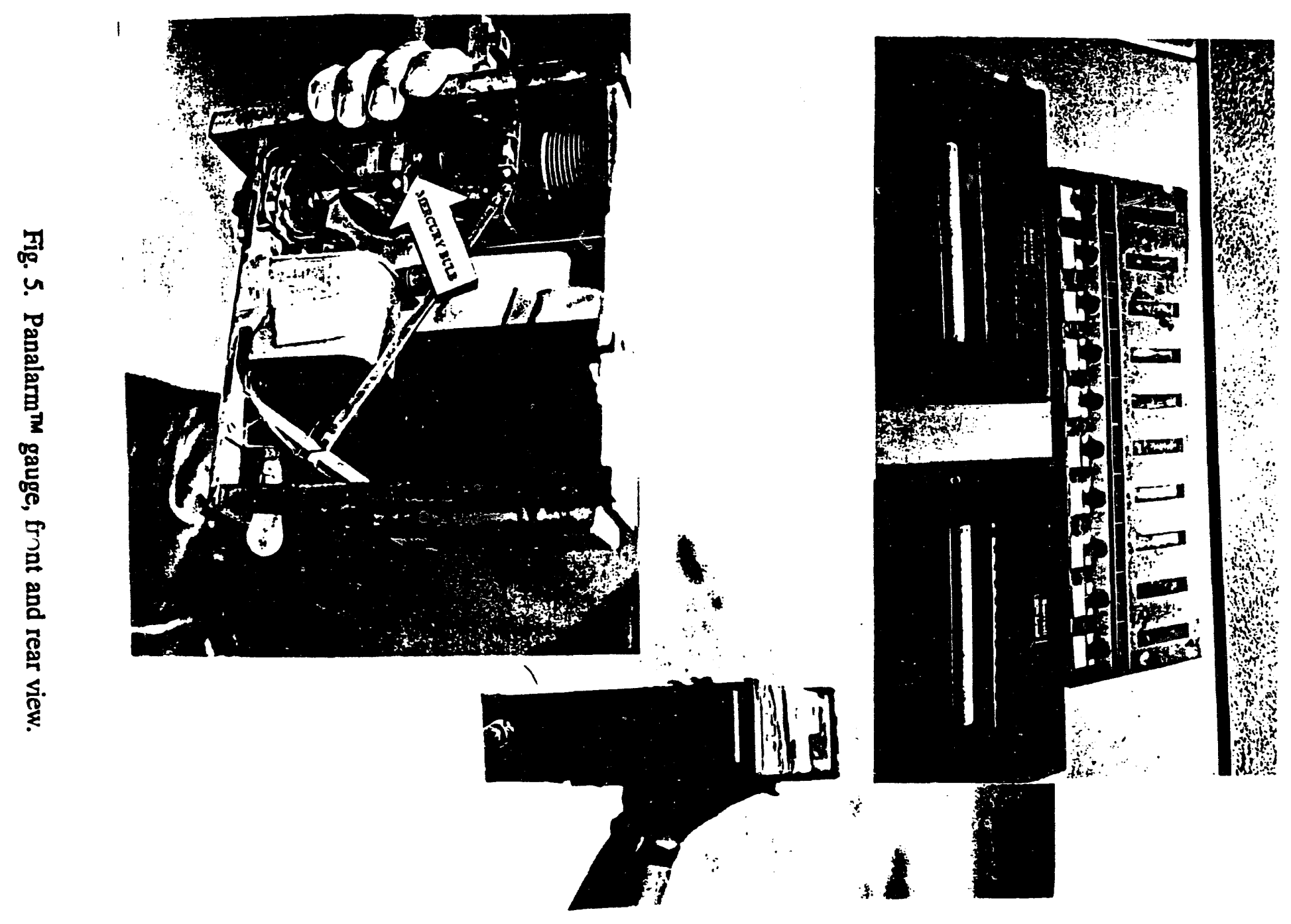




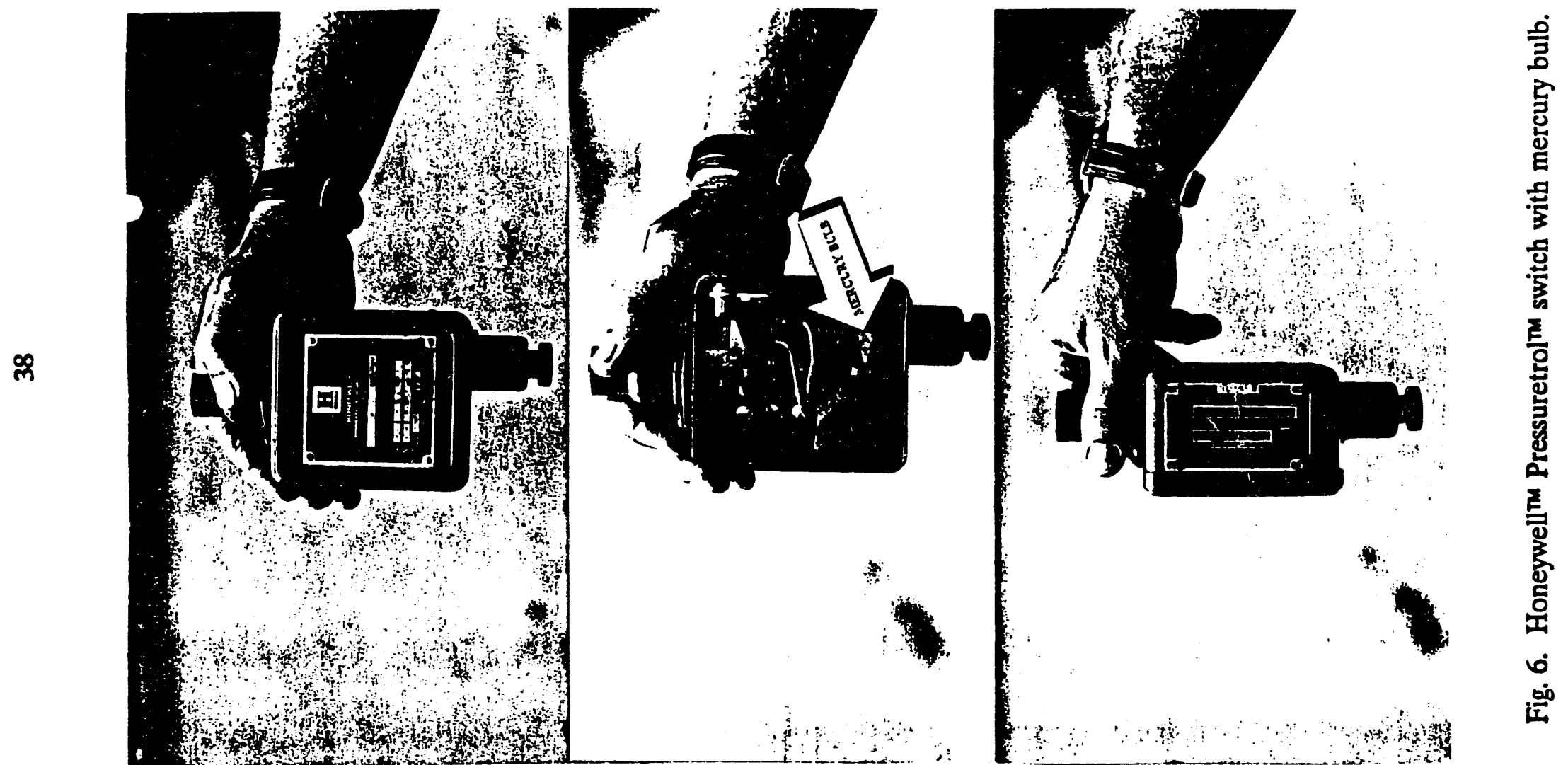




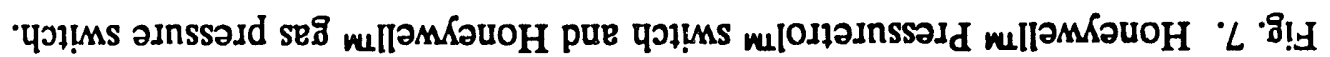
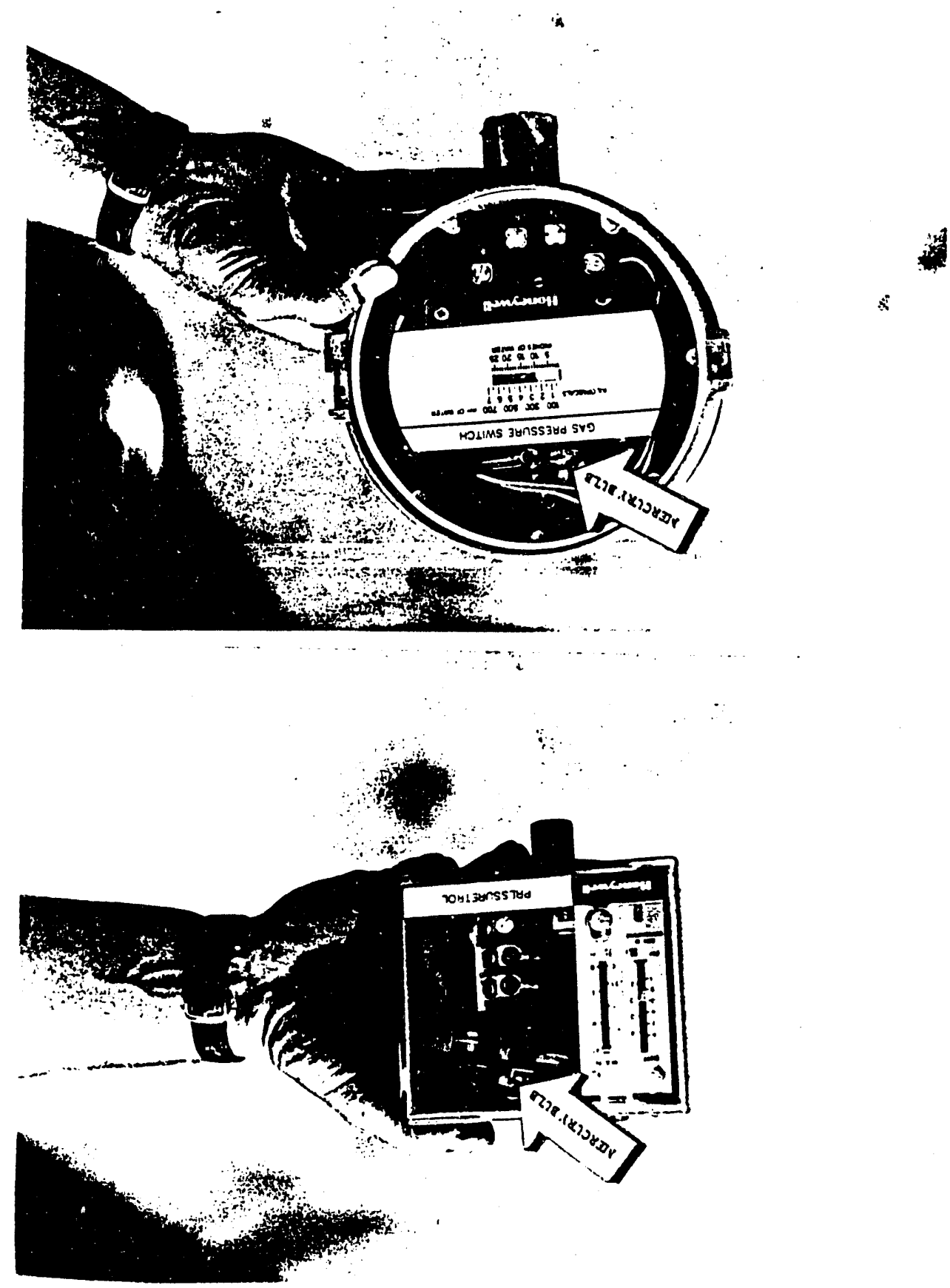


\section{HEALTH AND SAFETY ISSUES}

The primary health risks associated with mercury are skin absorption and inhalation of the vapor. Prolonged exposure to mercury vapor adversely affects the nervous system. Symptoms may include irritability, depression, vivid dreams, inflammation of the gums, insomnia, loss of memory and concentration, and constricted visual fields.

The OSHA PEL for mercury is $0.100 \mathrm{mg} / \mathrm{m}^{3}$ and the American Conference of Governmental Industrial Hygienists (ACGIH) threshold limit value (TLV) is $0.05 \mathrm{mg} / \mathrm{m}^{3}$. Both of these standards are based on an eight hour day (NIOSH 1985).

A site-specific and job-specific health and safety plan for the audit was prepared following the requirements of the PMRMA and the HAZWRAP health and safety plan (HAZWRAP 1990) and in accordance with OSHA requirements for hazardous waste site operations described in Title 29 of the Code of Federal Regulations, Part 1910.120. The health and safety plan established personal protection standards and mandatory safety practices for the mercury audit.

In order to mitigate the risk of exposure to chemical agents, the audit team gathered as much information as was available on the history of each building and interviewed PMRMA staff and subcontractors who had worked in the areas of concern. In most of the buildings, there was evidence that personnel had worked in or inspected the areas within a sufficiently recent period of time to conclude that risk to the audit team was minimal. Contact with surfaces suspected to be contaminated and walking through puddles and mud and on discolored surfaces were avoided.

Both audit team members were fit tested for RMA-issued gas masks; these accompanied each member throughout the surveys. A two-way radio obtained from the RMA Fire Prevention and Protection Branch accompanied the audit team in all survey areas except in the North Plants. A two-way radio obtained 
from a subcontractor, the Tennessee Valley Authority (TVA), was used in the North Plants. This provided the audit team with both information on TVA activities and a nearby emergency contact.

The audit team found RMA personnel and subcontractors to be extremely helpful with health and safety issues. Stearns and Rogers, another subcontractor, sent a representative to accompany the audit team in all buildings under their control. This proved to be quite useful, both in identifying health and safety concerns and locating mercury.

Access to buildings controlled by Weston, another subcontractor, required both reading and signing the Weston site-specific health and safety plan and a brief safety training. A representative of Weston accompanied the audit team and monitored the ambient air in buildings.

All access to the North Plant buildings was coordinated with the TVA. The list of buildings to be accessed was reviewed with TVA personnel, and special safety concerns were discussed. A representative from the TVA accompanied the audit team in Building 1501. Asbestos removal by Dominion, Inc., and dismantling of equipment by the TVA made this building a special safety concern. The TVA also was concerned about entry into Building 1611. An employee of the TVA had developed eye problems after entering this building the prior week. It was only after further investigation by TVA's industrial hygienist that the building was cleared for survey. The audit team exercised extreme caution in this building, wearing Tyvek $^{\mathrm{m}}$ suits, Sol-Vex gloves, boot covers, hard hats, and safety glasses.

All buildings that were part of the mercury audit were surveyed without incident. 


\section{REFERENCES}

HAZWRAP. 1990. Health and Safety Plan: HAZWRAP Support to the PMRMA at Rocky Mountain Arsenal. Hazardous Waste Remedial Actions Program, Martin Marietta Energy Systems, Inc., Oak Ridge, Tenn.

HAZWRAP. 1991. Program Management Plan for Environmental Support at Rocky Mountain Arsenal, Colorado. Revision 3. Hazardous Waste Remedial Actions Program, Martin Marietta Energy Systems, Inc., Oak Ridge, Tenn.

NIOSH. 1985. National Institute for Occupational Safety and Health Pocket Guide to Chemical Hazards. U. S. Department of Health and Human Services, Washington, D. C.

ORNL. 1992. Mercury Audit at Rocky Mountain Arsenal: Work Plan. Oak Ridge National Laboratory, Oak Ridge, Tenn. 


\section{ACRONYMS AND INITIALS}

\begin{tabular}{|c|c|c|}
\hline ACGIH & - & $\begin{array}{l}\text { American Conference of Governmental Industrial } \\
\text { Hygienists }\end{array}$ \\
\hline DOE & - & Department of Energy \\
\hline EPA & - & Environmental Protection Agency \\
\hline HAZWRAP & - & Hazardous Waste Remedial Actions Program \\
\hline NPL & - & National Priorities List \\
\hline ORNL & - & Oak Ridge National Laboratory \\
\hline OSHA & - & Occupational Safety and Health Administration \\
\hline PEL & - & permissible exposure limit \\
\hline PMRMA & - & Project Manager Rocky Mountain Arsenal \\
\hline RMA & - & Rocky Mountain Arsenal \\
\hline TLV & - & threshold limit value \\
\hline TVA & - & Tennessee Valley Authority \\
\hline
\end{tabular}


APPENDIX A

MERCURY AUDIT DATA BASE 


\section{A-1 \\ APPENDIX A \\ MERCURY AUDIT DATA BASE}

The data collected during the April-July 1992 mercury audit at the Rocky Mountain Arsenal was organized for display and transmission into a data base using KMan'M, a product of Micro Data Base Systems, Inc. The following is a description of the data base fields.

Field

No.

Name

Type

Length

Picture

1 Building or tank farm number

string

10

Tank group

string

10

3 Mercury survey date

string

8

$\mathrm{nn} / \mathrm{nn} / \mathrm{nn}$

4 Team members

string

36

$5 \quad$ Detection number

number

2

nn.

$6 \quad$ Mercury reading

number

5

n.nnn

7 Location of detection

string

30

8 Comments (memo field)

string

variable length 
All building and tank numbers (field No. 1) are sorted alphabetically. If no alphabetic characters exist at the beginning of these numbers, they are sorted numerically. A final alphabetic sort is conducted for numbers that end with alphabetic characters. Tank group numbers (field No. 2) are sorted alphabetically. Table 2.1, a listing of everything surveyed, is sorted as described above. Table 2.3, a listing of detections, is a subgroup of the data in Table 2.1 and is sorted similarly. Table 2.3 is created by the appearance of a number greater than zero in fields 5 and 6.

For the ASCII file sent to PMRMA, the database was sorted in report order. Each line of data contained the eight fields shown above, with no delimiters separating fields. Each line was up to 255 characters. When a record exceeded this length, the excess data was wrapped around the following line. 
ORNL/TM-12626

\title{
INTERNAL DISTRIBUTION
}
1. B. A. Berven
2. M. K. Jensen
3. N. E. Korte
4 - 9. C. A. Little
10. P. T. Owen
11. S. M Smith

\author{
12. Central Research Library \\ 13 - 14. Laboratory Records \\ 15. Laboratory Records - RC \\ 16. ORNL Patent Section \\ 17. ORNL Technical Library, Y-12
}

\section{EXTERNAL DISTRIBUTION}

18. G. M. Anderson, Program Management, Rocky Mountain Arsenal, Commerce City, CO 80022-1748

19. Maj. Wendall Moore, Program Management, Rocky Mountain Arsenal, Commerce City, CO 80022-1748

20. Office of Assistant Manager, Energy Research and Development, Oak Ridge Operations Office, P.O. Box 2001, Oak Ridge, TN 37831-8600

21 - 22. Office of Scientific and Technical Information, U.S. Department of Energy, P.O. Box 62, Oak Ridge, TN 3783 

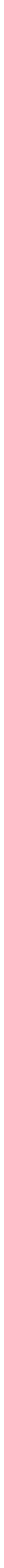
